\title{
PERSPECTIVES ON ALTERNATIVES TO PHTHALATE PLASTICIZED POLY(VINYL CHLORIDE) IN MEDICAL DEVICES APPLICATIONS
}

Federica Chiellini, ${ }^{1 *}$ Marcella Ferri, ${ }^{1}$ Andrea Morelli, ${ }^{1}$ Lucia Dipaola, ${ }^{3}$ Giuseppe Latini ${ }^{2,3}$

1. BIOlab, Department of Chemistry and Industrial Chemistry, University of Pisa, Via Risorgimento 35, 561267 Pisa, Italy

2. Division of Neonatology, Perrino Hospital, Brindisi, Italy

3. Clinical Physiology Institute, National Research Council of Italy (IFC-CNR), Italy

* Corresponding author. Tel:+39 050 2210305; fax: +39 050 2210332; e-mail address:

federica@dcci.unipi.it (Federica Chiellini)

Keywords: Poly(vinyl chloride), Phthalates, Plasticizers, Medical Devices

\begin{abstract}
Poly(vinyl chloride) (PVC) is one of the most important polymeric materials available today and is used to manufacture many items, ranging from packaging and toys to healthcare devices. PVC is per se a rigid material but it is made softer by compounding with plasticizers, particularly phthalate esters such as di-(2-ethylhexyl) phthalate (DEHP). In flexible plasticizer PVC (P-PVC), phthalates are not chemically bound to PVC and they are released into the external environment. In particular, prolonged contact of P-PVC based medical devices with body fluids or tissues has been shown to be associated with severe health risks. Major concerns regarding the safety of P-PVC in medical plastic items have been raised, and several alternatives to phthalates and to P-PVC itself as well as chemical/physical treatments of P-PVC to reduce DEHP migration have been proposed.
\end{abstract}


This review outlines recent scientific approaches for preventing DEHP contamination of humans by P-PVC medical devices, highlighting the impact of the proposed alternative materials on human health and strategies for implementing them. 


\section{Table of Contents}

\section{Abbreviations}

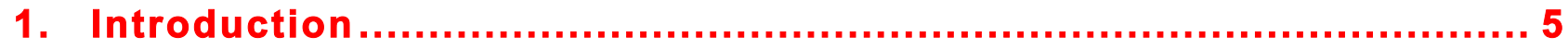

2. Alternatives to DEHP plasticized PVC .................................... 12

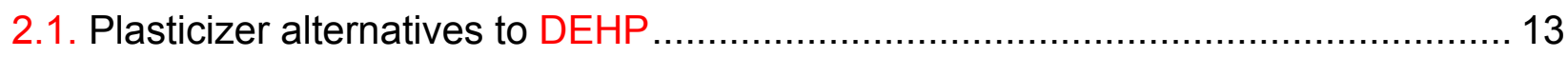

2.1.1 Low molecular weight plasticizers ................................ 15

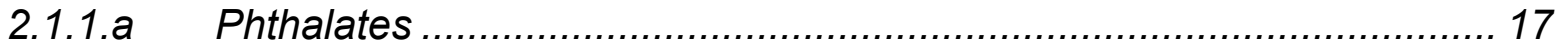

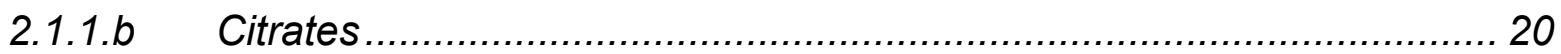

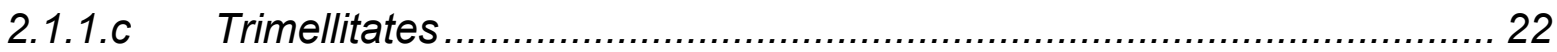

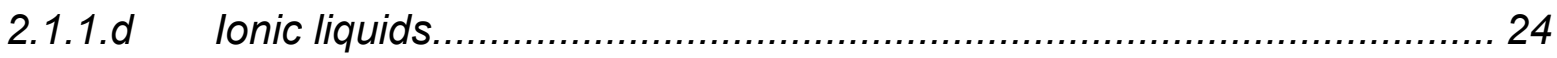

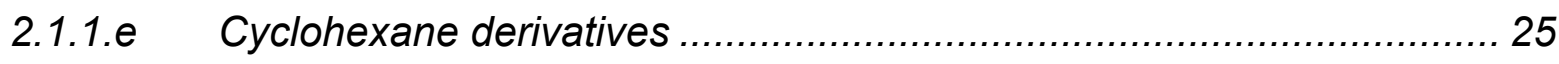

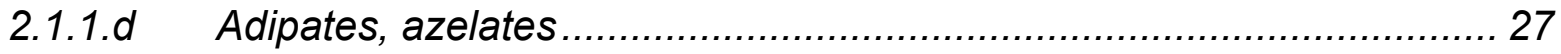

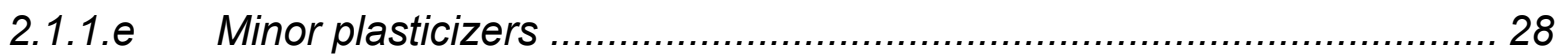

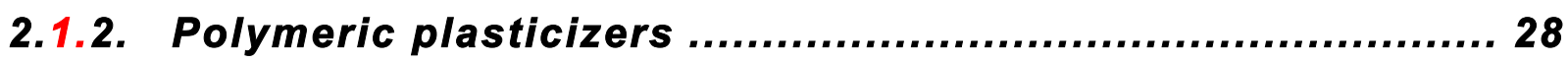

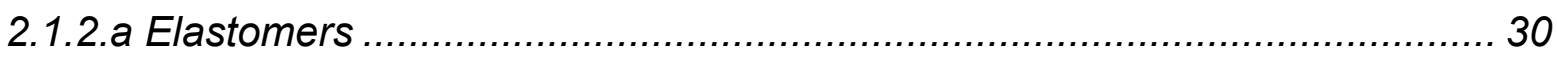

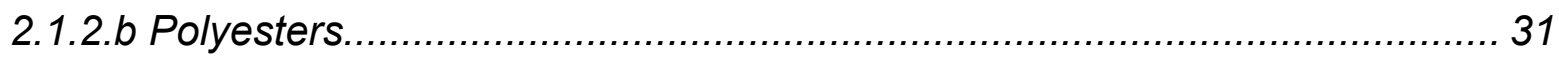

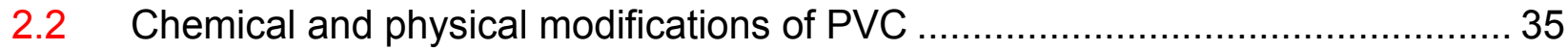

2.2.1. Surface Modification of PVC ......................................... 36

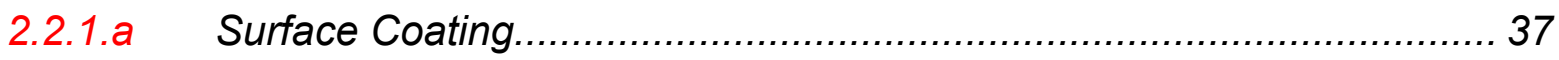


2.2.1.b. Surface crosslinking ......................................................... 40

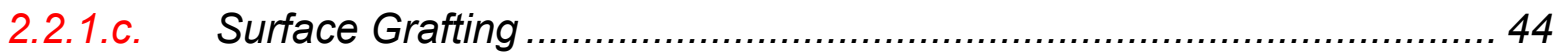

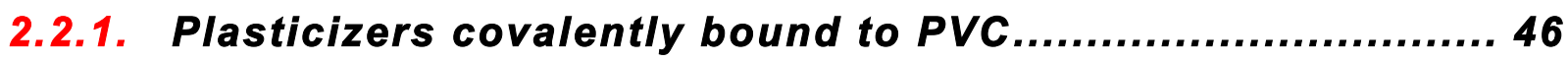

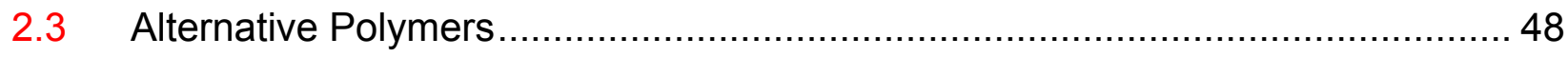

2.3.1 Ethylene/vinyl acetate copolymers (EVA) ...................... 49

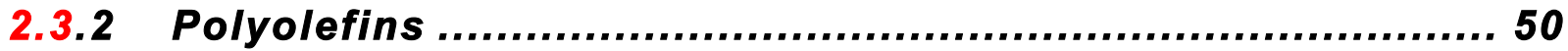

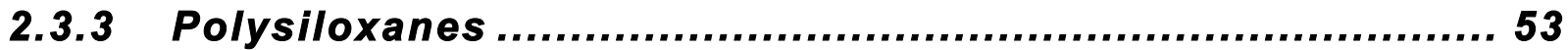

2.3.4 Polyurethanes ..................................................... 54

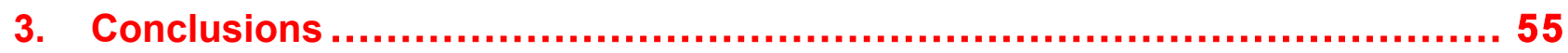


Abbreviations

Bz-â-CD 2,3,6-per-O-benzoyl-â-cyclodextrin

Citroflex A-4 Acetyltri-n-butyl citrate

Citroflex B-6 n-Butyryl-tri-n-hexyl citrate

CVD Chemical vapor deposition

DEHA Di(2-ethylhexyl) adipate

DINP Di-(isononyl phthalate)

DINCH Di(isononyl)-cyclohexane-1,2-dicarboxylate

DEHP Di-(2-ethylhexyl) phthalate

DEHT Di-(2-ethylhexyl) terephthalate

DOP-SH Di-(2-ethylhexyl) 4-mercaptophthalate

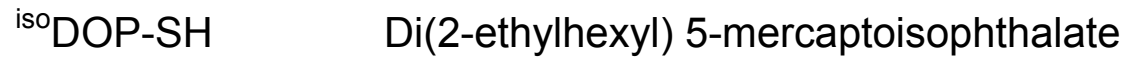

EPA Environmental Protection Agency

EVA Poly(ethylene-co-vinyl acetate)

GRAS Generally recognized as safe

IARC International Agency for Research on Cancer

ILs Ionic liquids

ISO International organization for standardization

LCA Life Cycle Analysis

MEHP Mono(2-ethylhexyl) phthalate

$\mathrm{NIOSH} \quad$ National Institute for Occupational Safety and Health 
OSHA

Occupational Safety and Health Administration

PVC

Poly(vinyl chloride)

PAA

Poly(acrylic acid)

PBA

Poly(butyl adipates)

PC

Poly(carbonate)

PCL

$\operatorname{Poly}(\varepsilon$-caprolactone $)$

PDMA

Poly(dimethyl acrylamide)

PDMAEMA

Poly(dimethylaminoethyl methacrylate)

PE

Polyethylene

PEG

Poly(ethylene glycol)

PEO

Poly(ethylene oxide)

PHEA

Poly(2-hydroxyethyl acrylate)

PHEMA

Poly(2-hydroxyethyl methacrylate)

POSS

Polyhedral oligomeric silsesquioxane

PP

Polypropylene

PPARa

Peroxisome proliferator-activated receptor alpha

PU

Poly(urethanes)

P4VP

Poly(4-vinylpiridine)

TOTM

Tris(2-ethylhexyl)trimellilate

TPN

Total parenteral nutrition 


\section{Introduction}

Poly(vinyl chloride) (PVC) is one of the most common thermoplastic materials employed today, with applications ranging from packaging to healthcare devices, toys, electrical wire insulation, clothes, furniture, packaging, interior decoration, building materials and the car industry [1]. PVC possesses the largest share of the medical market constituting $40 \%$ of all dedicated polymeric materials [2]. It is the first choice for medical applications due to its inertness, high transparency, facility of sterilization and strength. However, the inherent rigidity of PVC attested by its high glass transition temperature $\left(80^{\circ} \mathrm{C}\right)$ limits its applicability. To extend its versatility PVC is commonly compounded with different amounts of plasticizers and is commercially available with different grades of flexibility according to the end-user applications (Figure 1).

(Figure 1)

PVC can be manufactured in several grades, from rigid ( $70 \%$ market share) to soft and flexible (30\% market share). In biomedical applications PVC is mostly exploited as flexible PVC (P-PVC) whose plasticizer content may reach up to $50 \%$ of the total weight formulation [3]. Low cost and ease of manufacturing have led to the wide application of PPVC in single-use, sterile and disposable blood-contacting devices. About one-third of disposable medical devices are manufactured from P-PVC and it is the main thermoplastic 
material (85\%) in pre-sterilized single-use applications [4]. Disposability is a major advantage of P-PVC since the use of single-application medical devices is fundamental to reducing the occurrence of transfusion-transmitted diseases. P-PVC is mostly used in medical devices that interact, both directly or indirectly, with human body fluids or tissues (e.g., blood or intravenous storage bags and/or tubing). It is widely employed in the storage of blood derivatives due to the ascertained stabilizing effect exerted by this material on red blood cells [5]. The massive exploitation of P-PVC in crucial medical applications has raised concerns about the safety profile of this material and prompted scientists to accurately evaluate its toxicity. P-PVC toxicity could arise both from the polymer and the plasticizer since this material is commercially manufactured by compounding the polymer with a large amount of this additive (up to $50 \% \mathrm{w} / \mathrm{w}$ ). Polyvinyl chloride is considered inert and listed as non-carcinogenic by the most important environmental and occupational organizations (e.g. OSHA, NIOSH, NTP, IARC or EPA). The massive presence of plasticizer in P-PVC prompted scientists to consider the role of the additive as a critical key-point for the safety profile of the material and to accurately evaluate its effect on living organisms and environment.

The most widely used PVC plasticizers are phthalate esters, particularly di-(2-ethylhexyl) phthalate (DEHP), which usually represent up to $40-50 \%$ of the weight of the plastic items. Phthalates account for $80 \%$ of all plasticizer production [6] while DEHP is responsible for over $50 \%$ of worldwide phthalate production [7]. To date DEHP is regarded as the best additive for the plasticization and processability of PVC and its competitive costs led 
biomedical polymer manufacturers to exploit it extensively. A serious drawback of DEHP is its ascertained leachability from the medical P-PVC devices upon contact with body fluids or tissues [8-10]. The above shortcoming is worsened by the large amount of plasticizer usually present in the commercial material. To date the use of DEHP is somewhat regulated in medical applications but not forbidden, although many studies have proven its toxicity $[11,12]$. The labelling of products containing DEHP is now mandatory and its use, especially in the case of vulnerable users (e.g., long-term transfusion patients, male newborns, and pregnant women) is not recommended. The matter regarding DEHP toxicity in humans is still an open issue. To date DEHP is not considered carcinogenic to humans exclusively by indirect evidence, and its long-term effects are still uncertain. In laboratory animals (rodents and primates), both DEHP and its metabolite hemiester [mono(2-ethylhexyl) phthalate (MEHP)] produced toxic effects in multiple organ systems including the liver, reproductive tract (testes, ovaries), kidneys, lungs, and heart [11, 12]. The observed toxicity is assumed to be induced by DEHP and related metabolites through peroxisome proliferation. The above represents a threshold-based mechanism that leads to an uncontrollable activation of specific nuclear receptors (peroxisome proliferatoractivated receptor PPAR $\alpha$ ) that are physiologically involved in the regulation of lipid metabolism and glucose homeostasis [13]. The uncontrollable activation of this process is known to trigger liver cancer in rodents but its role is still uncertain in humans [14]. The possible lack of carcinogenicity of DEHP in humans is mostly supported by indirect evidence such as the lower expression of PPARa in human liver [15] and the differences in 
the receptor functions between rodents and mammals [14]. The debate is still open and requires additional study to clarify the mechanisms of interactions between DEHP and the host organisms. Peroxisome proliferation has not been established as the exclusive step in the toxicity of DEHP [16], and alternative routes for its carcinogenicity have been postulated $[16,17]$.

The few studies carried out on humans proved that DEHP exposure is associated with severe diseases such as bronchopulmonary dysplasia, cholestasis and deep venous thrombosis [18-21]. The above findings are of particular concern for vulnerable and longterm users. High-risk newborns undergoing intensive therapeutic medical interventions proved to be exposed to high concentrations of DEHP [22, 23], indicating that they are at the highest risk to adverse health outcomes after exposure to DEHP [24, 25]. A further study revealed that DEHP is released from P-PVC endotracheal tubes mostly during initial contact with the physiological fluids of the host organisms [10]. Accordingly, the recurrent applications of disposable P-PVC devices during intensive medical therapies would result in higher absorption of DEHP and might increase risks related to their use.

The questionable safety of DEHP and the consequent pressing call for its banning triggered scientists to investigate valid substitutes. The alternatives need to maintain the high performance characteristics of DEHP in plasticizing and processing PVC while not inducing adverse effects on living organisms. Phthalates containing different aliphatic substituents were scrutinized first due to their structural similarities with DEHP. Their safety profile is even more uncertain than that of DEHP since they have been studied less. 
On the whole, these chemicals cannot be claimed as valid alternatives to DEHP since their metabolism would likely follow similar pathways and their use is forbidden in other P-PVC applications (e.g., toys and baby-care products). However, a description of the most promising phthalates is reported in this review to provide an exhaustive perspective on the alternative plasticizers to DEHP.

The widespread use of P-PVC in crucial fields such as biomedical devices and childcontact items led to an upsurge of interest in finding new strategies for overcoming toxicity concerns. All the approaches are aimed at preventing the toxicity of the plasticizer since it is widely accepted as the exclusive cause of concern of P-PVC. To address this problem three main strategies have been pursued by researchers, namely the development of safe plasticizers alternative to DEHP, reduction of the leaching aptitude of plasticizers and the substitution of P-PVC with alternative safe polymers.

Here we present a comprehensive review of the currently available approaches, including their detailed descriptions and a critical evaluation of their feasibility in biomedical applications. However, identifying the best alternatives would require an exhaustive assessment of their advantages and drawbacks through a detailed life-cycle analysis (LCA). The LCA would provide valid criteria to determine the environmental impacts and costs of the envisaged approaches and an overall perspective of their risks and benefits from the extraction of the raw materials to the final disposal of the end products. Such a complex analysis still lies beyond the aim of this review, whose scope is to offer a 
perspective on the feasibility of current approaches in solving P-PVC concerns in the medical field.

\section{Alternatives to DEHP plasticized PVC}

At present different strategies have been used to develop a material alternative to the conventional DEHP plasticized PVC (Figure 2).

(Figure 2)

All the approaches converged to limit the harmful effects of the plasticizer either by improving its safety or by preventing its release into physiological fluids. The replacement of PVC with suitable alternative materials is taken into account as a valid opportunity to avoid the use of plasticizers. This strategy is particularly attractive due to the combined possibility of using a safe polymer without any added plasticizer, but it is highly challenging since PVC is still one of the best biomaterials in terms of inertness, cost and function. Different strategies have been undertaken to limit the plasticizer concerns and extend the use of PVC in medical applications. The use of DEHP is still envisaged due to its high performing properties in terms of plasticization and processing of the polymeric matrix. Accordingly, researchers investigated the chemical and physical modification of PVC as a valuable strategy to definitely entrap DEHP into the PVC matrix, thus drastically reducing 
its release upon contact with biological fluids. The research for alternative plasticizers to DEHP is one of the most currently investigated approaches to overcoming the P-PVC concerns, but the main challenge is identifying plasticizers having competitive all-around performance comparable to that of DEHP.

In general, any valid alternative should fulfil the essential regulatory requirements and have a competitive all-round performance comparable to that of P-PVC. A brief survey of the advantages and drawbacks of the currently investigated approaches is reported in Table 1. A more comprehensive discussion of their feasibility in the substitution of conventional P-PVC formulations will be debated in the following paragraphs.

(Table 1)

\subsection{Plasticizer alternatives to DEHP}

The use of PVC in medical devices is still widespread due to its cheapness, ease of manufacturing and inertness. Its future exploitation has been exclusively questioned due to the toxicity and leaching aptitude of the plasticizer used in commercial P-PVC formulations. The research of alternative plasticizers with favorable toxicity profiles would be a valid strategy for extending the use of PVC in medical applications. Biocompatibility of the plasticizer and any relevant degradation products are an indispensable requirement for safely fulfilling the crucial applications of P-PVC. However, biocompatibility is not the exclusive criterion for a suitable substitution of DEHP in P-PVC. The overall performance of DEHP in terms of plasticization and processability of the polymer should be properly 
matched in order to maintain the advantages of conventional P-PVC. A valid alternative to DEHP should also be suitable for maintaining the mechanical properties of the material during its entire working life. Most plasticized materials typically lose their flexibility upon recurrent application due to the volatilization and leaching of the plasticizer into the nearby environment. Performance of P-PVC is greatly affected by the above phenomena due to its large plasticizer content and the leaching aptitude of DEHP. The loss of plasticizer is a cause for concern, especially in medical applications where the persistent flexibility of the device material is strictly required for encouraging patient compliance (e.g., catheter care). To address this concern and reduce P-PVC toxicity, research has been focusing on the development of novel plasticizers with reduced leaching aptitude. However the leaching behavior of the plasticizer is strongly affected by the chemical nature of the surrounding liquid and cannot be universally optimized for each application. Most of the candidates investigated are of synthetic origin and their safety profile has been underestimated, especially regarding long-term periods. Exploiting plasticizers of natural origin would somewhat overcome concerns related to their biocompatibility but nowadays it is hampered by economic reasons. Waste and renewable resources could supply cheap and safe plasticizers, as the best solution to P-PVC concerns. The following paragraphs report several classes of chemicals that have been investigated as potential alternatives to DEHP in medical devices. The candidates are classified according to their molecular weight as (a) low molecular weight plasticizers and (b) polymeric plasticizers. 


\subsubsection{Low molecular weight plasticizers}

Low molecular weight PVC plasticizers such as citrates, adipates, trimellitates, azelates, sebacates etc (Figure 3), are currently under investigation and are slowly conquering market shares traditionally pertaining to DEHP. However, their overall consumption is still only a small fraction of the total consumption of phthalates.

(Figure 3)

The main drawback of low molecular weight additives is their tendency to leach from the polymer matrix. The loss of plasticizer occurs typically by a two-step mechanism, namely migration of the additive to the interface with the medium and subsequent dissolution. Parameters such as polarity of the medium and plasticizer and the affinity between the plasticizer and the polymer strongly affect the rate of leaching. Normally, a good PVC plasticizer should contain both polar and non-polar fragments. The polar groups are needed to provide affinity for the polymer while the non-polar part is responsible for plasticization [6]. The hydrophobic part commonly constitutes the major fraction of a valid PVC plasticizer. The leaching behaviour of the plasticizer cannot be universally predicted since it depends on the nature of the surrounding media as well. In aqueous medium, the dissolution of the plasticizer is the rate-determining step of the leaching process. In more non-polar media the diffusivity of the plasticizer in the polymer matrix mostly controls its leaching rates [26]. As a consequence, when a plasticizer is investigated as a suitable 
alternative, considerations must include the plasticizer's compatibility with PVC, its tendency to leach into the surrounding media, the amount of plasticizer required to achieve the desired flexibility, and its toxicity in terms of accumulation risk and its long-term effects on humans. The critical applications of P-PVC would require additional research towards the assessment of biocompatibility of the additives. To date the safety profiles of all the investigated plasticizers are still ambiguous, since most of the research has been focused on DEHP. The lack of toxicological data especially referring to long-term effects and human exposure is shown in Table 2.

(Table 2)

The ambiguous toxicological profiles of the selected plasticizers require careful consideration to establish whether they can be envisaged as Generally Recognized As Safe (GRAS) products in medical devices. To date their behavior can be mostly predicted indirectly based on their chemical structure and the knowledge of the mechanisms of leaching and toxicity in animal models. The currently available toxicological data will be reported in the following paragraphs to provide a more objective tool for comparative evaluation. 


\subsection{1.a Phthalates}

The excellent performances of DEHP in the plasticization and processing of PVC prompted scientists to first investigate the class of phthalates as a resource of alternative plasticizers. The strategy was to adjust the chemical nature of the lateral alkyl chains in order to reduce the leaching of the plasticizers into the surrounding medium. Major concerns persist and are related to the toxicological profile of these additives, since their metabolism would likely be similar to that of DEHP. Indeed, current legislation strictly limits the use of phthalates in crucial applications such as toys and child-care products. High molecular weight phthalates such as di-isononyl phthalate (DINP), di-isodecyl phthalate (DIDP) and di-n-octyl phthalate (DNOP) are allowed at concentrations lower than $0.1 \%$ by mass of the plasticized material only in children's articles that cannot be placed in the mouth. Low molecular weight phthalates including (2-ethylhexyl) phthalate (DEHP), di-nbutyl phthalate (DBP) and butylbenzyl phthalate (BBP) are no longer allowed as plasticizers in any kind of toys or childcare articles.

In the medical device market, two of the main alternatives to DEHP are the phthalate derivatives di-(isononyl phthalate) (DINP) and di-(2-ethylhexyl) terephthalate (DEHT).

DINP is a mixture of isomers that have alkyl chains from eight to ten carbons long, but the largest portion of the mixture has chains composed of nine carbons in various isomeric configurations. It is basically used as a plasticizer in PVC toys for children, but currently it is widely used in the production of medical tubing devices. 
Only a limited number of studies regarding the toxicological aspect of DINP are available [27-30], showing a toxicity profile similar to that of DEHP, but at higher exposure levels. No adverse effects have been reported on the fertility of animals. Rodents exposed to high doses of DINP were reported to suffer from skeletal and genitourinary abnormalities during embryo-fetal development [29]. In chronic/carcinogenicity studies with DINP, prolonged administration of the plasticizer caused significant increases in liver tumors in rats and mice, but no peroxisome proliferation in the liver of monkeys was observed.

DEHT is an isomer of DEHP based on terephthalic acid (Figure 3). The structural differences from DEHP (the two carboxylic groups of DEHT are located in the paraposition while in DEHP they are located in ortho-position) have important implications for the toxicological profile of the compound [31]. The different behavior is attributed to their different metabolic end-products. In vitro and in vivo studies of DEHT metabolism showed the complete hydrolysis of both ester linkages to two non-toxic products: 2-ethylhexanol and terephthalic acid [32]. In the case of DEHP only a partial hydrolysis of the ester linkage occurs and the formation of its metabolite MEHP is observed. Several studies have shown that many of the toxicological effects of DEHP are induced by MEHP [33-36]. Recently, the toxicity of DEHT in terms of acute, geno, subchronic, chronic, reproductive and developmental toxicity has been assessed [31, 36-39]. These studies showed low acute toxicity by oral route in rats with $L_{50}$ reported at $>5000 \mathrm{mg} / \mathrm{kg}$ for male rats and $5000 \mathrm{mg} / \mathrm{kg}$ for male mice [40]. Dermal LD $_{50}$ in male guinea pigs was found to be $>19670$ 
$\mathrm{mg} / \mathrm{kg}$ [39]. Slight dermal irritation in guinea pigs was observed, while no skin irritation was detected in humans and guinea pigs.

Deyo et al. [31] investigated chronic toxicity and carcinogenicity induced by dietary exposure to DEHT for 2 years at levels up to 12,000 ppm in Sprague-Dawley rats. The authors found that DEHT had no tendency to induce tumors and it showed low levels of toxicity. Developmental and reproductive toxicity of DEHT were also evaluated in Sprague-Dawley rats [36-38]. The studies showed no evidence of teratogenesis in the investigated species following dietary exposure to dose levels of DEHT between 747 and $1382 \mathrm{mg} / \mathrm{hg} /$ day.

In order to evaluate DEHT reproductive toxicity, a two-generation test was performed [38]. Results showed that the consumption of diets containing up to $1.0 \%$ DEHT did not affect male or female fertility in animals over two generations. Moreover, a preliminary human study on dermal application of DEHT showed no skin irritation or sensitization under the adopted experimental conditions [27].

According to the above findings and considering the competitive cost of DEHT [41] this plasticizer can be considered one of most promising alternatives among phthalates. However, its use in medical devices should be carefully considered due to rising worry about the adverse effects of phthalates. 


\subsection{1.b Citrates}

Citrate derivatives are an appealing class of plasticizers since they are obtained from citric acid, a common metabolite of plants and animals. Citrates are relatively expensive and do not display any outstanding technical advantages over phthalate plasticizers. However, their natural origin and overall fair performances prompted scientists to assess their feasibility in the substitution for DEHP in P-PVC. Indeed, they have been available for years as plasticizers in medical devices, especially for blood storage bags. Due to their unique aliphatic structure containing three carboxylic functional sites available for esterification, a wide variety of citrate esters have been produced. In this review we will focus on three citrate esters that have been widely used in the production of PVC DEHPfree medical devices under the trade name of Citroflex (Figure 3).

Citroflex A-4, an acetyltri-n-butyl citrate (ATBC), consists of citrate with three ester bonded butyl groups and one acetyl group bonded to the tertiary hydroxyl group (Figure 3). It is currently employed in aqueous pharmaceutical coatings and extra-corporeal tubing production [42]. Safety assessment of ATBC provided by the U.S. EPA HPV program [42] indicated that it did not induce adverse toxicological effects on animals. ATBC showed low acute toxicity by oral route in rats and mice, with $\mathrm{LD}_{50}$ values $>30$ and $>50 \mathrm{~mL} / \mathrm{kg}$, respectively. Repeated dose toxicity studies in rats and cats conducted for a period ranging from 14 days to 2 years showed low levels of subchronic toxicity. No adverse responses were obtained from genotoxicity, reproductive and developmental toxicity tests as well [43] and a low level of cytotoxicity was induced by ATBC in HeLa cell cultures [43]. 
A comparison between the toxicity of ATBC and DEHP in animals has been described in the report by SCENIHR on "The safety of medical devices containing DEHP plasticized PVC or other plasticizers on neonates and other groups possibly at risk" [27]. The authors found that ATBC showed a "No Adverse Effect Level (NOAEL)" value 20 times higher in comparison to DEHP. One of the major drawbacks of ATBC is its ease of leachability from PVC. The leaching rates of ATBC and DEHP from PVC items were compared by interfacing them with different feeding solutions [27]. ATBC showed a leaching rate ten times higher than DEHP, thus posing some concerns about its use in recurrent long-term applications.

Citroflex A-6 is an acetyl-tri-n-hexyl citrate with three ester bonded hexyl groups and one acetyl group bonded to the fourth available oxygen atom (Figure 3). Preliminary studies were undertaken by Hull et al. [44, 45] in order to provide a new citrate derivate with improved properties in terms of toxicity and leachability. The authors evidenced a reduced leaching of this plasticizer into various mediums and a low acute toxicity. It proved to cause no skin and ocular irritation and its oral administration produced no signs of systemic toxicity, and no adverse responses were evidenced from genotoxicity assays.

Citroflex B-6, n-butyryl-tri-n-hexyl citrate (BTHC), is a citrate with three ester bonded hexyl groups and one butyryl group bonded to the tertiary hydroxyl group (Figure 3). It represents the major alternative plasticizer to DEHP in the production of blood storage containers. Studies have suggested that transfusing platelets stored in BTHC containing bags is safe [46]. Its leachability into plasma is much lower than that of DEHP and in 
contrast to DEHP, it is metabolized to physiologic compounds as citric acid, butyric acid and hexanol.

The toxicological aspects of this plasticizer have been recently reviewed by SCENIHR [27].

Toxicology testing has shown that BTHC has low acute toxicity and a low irritation and sensitization potential. No mutagenic or genotoxic effects were observed in several bacterial tests, either with or without the presence of a metabolic activation system [27]. Citrate-based plasticizers could be a promising alternative to DEHP in view of their inherent biocompatibility. The optimization of their chemical structure as in the case of BTCH could overcome their leaching aptitude and possibly improve their technical performance as well. In absence of valid alternatives, the higher costs related to the use of citrates in P-PVC could be overcome by their use in added-value applications, such as in biomedical items.

\subsection{1.c Trimellitates}

Tris (2-ethylhexyl) trimellitate (TOTM), an ester of trimellitic acid, is commonly used as a plasticizer in medical devices in Japan [47]. This material is produced by the esterification of octyl alcohols with trimellitic anhydride (TMA), which shares a structure similar to that of phthalic acid with the exception of a third carboxylic functionality on the aromatic ring (Figure 3). This difference in chemical structure from that of DEHP leads to 
significant differences in terms of extraction and migration resistance of the material [27, $47,48]$.

Kambia et al. [48] evidenced a lower leaching of plasticizers from new hemodialysis tubing plasticized with the combination of TOTM-DEHP in comparison to DEHP alone. The leachability of TOTM from PVC tubes was also investigated by lto et al., by interfacing them with different intravenous preparations [47]. The authors described a new method for the determination of TOTM released from PVC medical devices based on liquid chromatography-tandem mass spectrometry. The results showed that the amount of TOTM released into lipophilic intravenous preparations was approximately 10 times lower than that of DEHP. The effects of gamma-ray sterilization on TOTM P-PVC were also investigated, indicating a lower release of plasticizer in comparison to the un-sterilized one. A similar behavior was observed by the same authors in the case of PVC-DEHP tubes. The formation of degradation products upon gamma irradiation was found to depend on the plasticizer used. A considerable production of MEHP was observed during the sterilization of DEHP P-PVC tubes compared to the unsterilized control $[49,50]$. The absence of degradation products was revealed by analysis of TOTM plasticized PVC samples indicating the resistance of the additive to irradiation [47].

Very little information is available about the toxicity of TOTM. It has been reported that TOTM was estrogenic in both estrogen receptor alpha and beta cells [51] and showed weaker hepatoxicity than DEHP [52]. David et al. [53] carried out a human repeated insult patch test in order to determine whether alternative plasticizers to DEHP pose undue risk 
for human sensitization. The results pointed out that TOTM displayed low potential for sensitization in humans and that it could be a suitable substitute for o-phthalate. However, according to Kambia's criticism [48], additional studies on TOTM would be required focusing on the toxicity, metabolism and long-term effects on humans. New generation PPVC include PVC plasticized with TOTM since this plasticizer is reported to leach from the plastic to a lesser extent in comparison to DEHP, most likely due to its higher molecular weight [3]. A prominent advantage of TOTM P-PVC materials is their improved gas exchange capacity, which is beneficial for platelet survival [3]. For this reason DEHP has been almost fully replaced by TOTM in the plasticization of bags devoted to platelet storage [3]. TOTM proved to be unsuitable for red cell storage, while DEHP and citrates have been shown to have a stabilizing effect on red blood cell membranes [3]. The above findings led to the formulation of different P-PVC materials according to the applications envisaged.

Trimellitates are a promising class of plasticizers in terms of overall technical performance, closely matching that of DEHP [26]. However, their chemical resemblance to DEHP poses some concerns regarding their toxicological profile.

\subsection{1.d Ionic liquids}

Ionic liquids (ILs) were recently suggested as alternative plasticizers in PVC formulations [54, 55] (Figure 3). ILs are salts in which the ions are poorly coordinated and 
as a consequence these solvents are liquid below $100^{\circ} \mathrm{C}$, or even at room temperature (room temperature ionic liquids, RTIL's).

It has been found that several ILs, based on ammonium, imidazolium and phosphonium actions, did impart significant flexibility to PVC and showed better leaching and migration resistance than the traditional plasticizers [55]. Studies of leaching carried out on PVC-ILs plasticized items upon UV irradiation did not evidence any weight loss and exudation of the plasticizer [55].

To date ILs have not been introduced on the medical market, due to the high cost of the materials and the overall lack of data regarding their potential toxicity.

\subsection{1.e Cyclohexane derivatives}

A promising alternative plasticizer is currently produced by BASF under the trade name of Hexamoll DINCH. This plasticizer is a di(isononyl)-cyclohexane-1,2-dicarboxylate (Figure 3) obtained by substitution of the benzene ring of DEHP with a cyclohexane ring. This substitution strongly affects the properties of the derived plasticizer both for electronic and sterical reasons. In a comparative study between different plasticizer added to PVC the leaching behavior of DINCH was found to be far less pronounced [56]. By comparing the leaching behavior in different feeding solutions, DINCH showed a tendency to migrate that was three to ten times lower than that recorded for DEHP [27].

The higher leaching rate of DEHP can be attributed to the higher polarity induced by the aromatic ring on the molecule that would eventually promote its dissolution in the aqueous 
media. A prominent advantage of $\mathrm{DINCH}$ is related to its physical behavior, similar to that displayed by DEHP. In a comparative study [57], the authors showed that the two plasticizers have similar viscosities and suggested that the relevant P-PVC formulations would not require significant changes in the plasticizer content and viscosity modifiers. This makes processing P-PVC with existing equipment potentially feasible.

DINCH was recently introduced on the market and it is suggested as an alternative to DEHP for the production of blood tubes and packaging for nutrient solutions. With this aim, a formulation based on commercial PVC resin plasticized with DINCH was subjected to repeated sterilization processes by contact with ethylene oxide (EO) at low pressure and temperature and (ii) water vapor with $2 \mathrm{wt} \%$ of formaldehyde and subsequently analyzed [58]. The effects of the sterilization processes were thoroughly investigated since the above treatments could cause the loss of the mechanical and physical properties of the materials and the formation of products of degradation detrimental for patient compliance $[49,59-61]$. The results evidenced only slight structural modifications of the analyzed samples and insignificant surface modification, without any proof of serious degradation. Some toxicological aspects of DINCH were provided by the manufacturer [62] and recently reviewed by SCHENIR [27]. Data revealed that DINCH induced very low acute toxicity in rats after oral and dermal administration and a 28-day feeding study gave no indication of effects on the testicles or ovaries. Studies on prenatal development and two-generation effects were carried out as well and the results showed that DINCH did not induce any reproductive toxicity. Exposure to DINCH was not found to induce mutagenicity or 
genotoxicity. An increased thyroid weight in rats was observed in a 2-year combined chronic toxicity/carcinogenicity study, but the effect was interpreted as due to the secondary mechanisms via liver enzyme induction, which is considered not relevant for humans.

To date DINCH could be considered the most promising candidate for the substitution of DEHP in P-PVC. It has been shown to have low environmental persistence and high biodegradability compared to DEHP, and the preliminary toxicity tests are promising [62]. Accordingly, the above findings merit further confirmation, especially if carried out by independent research groups, in order to elect DINCH as the best alternative to DEHP.

\subsection{1.d Adipates, azelates}

These classes of plasticizers are characterized by a similar chemical structure generally composed by a linear aliphatic chain terminated with two carboxylic ester groups (Figure 3). 2-ethyl hexyl alcohol is the chemical most often used for the esterification of the commercial plasticizers. Their low inherent viscosity given by their flexible linear molecular structure provide them with good low-temperature plasticizing performance [26]. Adipates have been historically exploited as plasticizers of PVC in food packaging applications [3] Their higher extractability in comparison with DEHP mainly hampered their use in medical P-PVC [3]. The use of azelates could overcome the leaching concerns related to adipates since they contain a more hydrophobic aliphatic core but their exploitation in medical PPVC is limited to date. A recent study carried out on the toxicological profiles of selected 
plasticizers revealed that some metabolites of the most representative adipate (di(2ethylhexyl) adipate (DEHA)) could trigger peroxisome proliferation as in the case of DEHP [17]. The above finding poses some concern about the possible future exploitation of this class of plasticizers in medical P-PVC.

\subsection{1.e Minor plasticizers}

In addition to the low molecular weight plasticizers described so far, studies in literature report the possible use of benzoate ester [63] and of ester of aconitic acid [64] as alternative PVC plasticizers. However, no toxicological properties have been reported so far and only preliminary data regarding their thermal and mechanical properties are available in literature $[57,58]$.

\subsubsection{Polymeric plasticizers}

A promising and appealing way to obtain safe plasticized PVC grades is to substitute phthalates and low molecular weight plasticizers, with analogous compatible and non-toxic polymeric plasticizers, thus generating a kind of "polymeric alloy". Due to their molecular dimensions and intrinsic compatibility, the polymeric plasticizer shows no tendency to de-mix and migrate to the surface of PVC products. Leaching and volatility issues have been significantly improved over traditional plasticizers and the flexibility of the blended plastics lasted for extended lifetimes [26]. 
Major drawbacks of these plasticizers are their low performance in terms of processing and mixing with the polymer resin and a reduced plasticizing efficiency with respect to the low molecular weight counterpart. The increase in tensile strength and reduced elongation at break are usually reported for this kind of "polymeric alloy", thus confirming a general stiffening upon formation [65]. While polymeric plasticizers may cause reduced flexibility in plastic materials, they can also be used in combination with traditional plasticizers to improve leaching resistance of the latter. The molecular weight of the polymeric plasticizer deeply affects both the flexibility of the polymeric matrix and the diffusivity of additionally incorporated additives. Polymeric plasticizers with higher molecular weight would impart less flexibility to the matrix but result in exceptionally good resistance to extraction, migration and volatile loss [3].

Figure 4 reports the structures of the most common polymeric plasticizers used both in combination with DEHP and as a unique plasticizer in PVC products.

\section{(Figure 4)}

All the investigated polymeric plasticizers suffer from lack of toxicological data referring to their use in medical P-PVC. The absence of leaching usually found during the experiments does not justify the above deficiency since the plasticizers could degrade during their exposure in the working media. The products of degradation of the polymeric plasticizers could cause some concerns and their assessment would be worth further investigation. 
The low grade of plasticization of these additives would require a greater amount of material to achieve the same performance as DEHP, thus increasing the risk related to their use. The exploitation of biocompatible polymeric additives whose products of degradation are known to be safe (e.g., polycaprolactone or polyhydroxyalkanoates) could efficiently overcome the problem.

\subsection{2.a Elastomers}

The use of elastomers aimed to overcome the limited plasticization efficiency typical of polymeric plasticizers. Three elastomers, namely nitrile rubber, carboxylated nitrile rubber and epoxidized natural rubber, have been evaluated as plasticizers of P-PVC in combination with DEHP [66]. The study evidenced a decrease in the DEHP migration from PVC correlated to the amount and nature of the elastomers employed. Nitrile rubber was found to provide greater resistance to leaching of DEHP. Overall the study showed that migration of the DEHP can be reduced significantly without affecting the useful mechanical properties of the polymer [66].

Polysiloxane plasticizers could be conventionally classified as elastomeric materials. Oligomeric polysiloxanes could impart flexibility to PVC due to their inherent physical properties and low molecular weight. Polyhedral oligomeric silsesquioxane (POSS), a hybrid organic-inorganic compound (Figure 4), was investigated in combination with DEHP for the plasticization of PVC [67]. POSS consists of a silica cage in the center with functional organic groups attached at the various cage corners. Due to its hybrid nature, 
POSS is more stable and less volatile in comparison to low molecular weight plasticizers. The introduction of methacryloyl groups in the POSS structure promotes its covalent conjugation to PVC by radical grafting reactions, thus further minimizing possible leaching from the matrix. The methacryloyl moieties could also increase the compatibility between the siloxane and PVC and the affinity with DEHP. However methacryloyl-POSS was found to have limited miscibility with PVC, up to $15 \% \mathrm{wt}$, and as a consequence its use alone was precluded. The authors showed that the addition of a small amount of DEHP (5 wt\%) substantially increases the miscibility of methacryloyl-POSS in PVC. It was found that POSS/DEHP-plasticized PVC had different mechanical behavior in low rate compression and tensile experiments when compared to the DEHP-plasticized PVC with the same glass transition temperature $(\mathrm{Tg})$. This result indicated the possibility of employing POSS to modulate the mechanical properties of plasticized PVC compounds, but the POSS/DEHP-plasticized PVC showed no ability to retain the plasticizers in the compound.

\subsection{2.b Polyesters}

Saturated polyesters constitute the class of polymeric plasticizers mostly investigated nowadays for the substitution of DEHP in P-PVC. Their compatibility with PVC is provided mostly by hydrogen bonding interactions between the carboxyl ester group and the hydrogen atoms in $\alpha$ or $\beta$ position with respect to the chlorine atom in PVC. The affinity between the two polymers is also enhanced by dipole-dipole interactions between the carboxyl ester group and the chlorine atom in PVC [68]. 
At present polyadipates (Figure 4) are the exclusive polymeric plasticizers employed in the medical device market. Since the early 1980s they have been used as plasticizers in feeding tubes and have been approved by healthcare authorities. Commercial PVC-polyadipate tubes, used for feeding infants in the hospital, have undergone aging tests and showed that polyadipate was stable in different working conditions [69]. Recently PVC nasogastric tubes containing polyadipate were proposed as an alternative to conventional devices for short-term applications [70]. Extraction experiments of DEHP and polyadipate from the samples have shown that leaching of polyadipate was 10 times lower than that recorded for DEHP in the feeding solution and 100 times lower in the gastric juice.

Poly( $\varepsilon$-caprolactone) $(\mathrm{PCL})$ and relevant copolymers have been proposed as valid alternatives to phthalate plasticizers due to their biocompatibility. Penco et al. [71] suggested the use of multi-block copolymers containing poly( $\varepsilon$-caprolactone) and poly(ethylene glycol) (PEG) segments (Figure 4). The study investigated the miscibility between PVC and the multi-block copolymers and the degree of compatibility necessary for plasticization. Results showed that the presence of poly( $\varepsilon$-caprolactone) blocks increases the miscibility of the PEG segments in the PVC matrix. A more detailed study was carried out by Ferruti et al. [72] within the framework of a European Project. The performance of a wide PCL-PEG copolymer series has been investigated, mainly focusing on dynamic mechanical analysis (DMA) and extraction experiments in aqueous media. The authors proved that copolymers with the shortest PEG segments, as well as PCL 
oligomers, had good miscibility with PVC and negligible tendency to be extracted by aqueous media. The authors interpreted the promising performances of the analyzed copolymers by considering the positive cooperative effects of the two polymeric blocks. The PCL blocks provided compatibility with PVC through hydrophobic interactions while PEG provided hydrophilic segments with favorable interactions with human tissue. More importantly, both polymers have been extensively employed in biomedical and pharmaceutical applications and are well known to be non-toxic materials. The authors concluded that both PCL oligomer and multiblock copolymers of PCL with low molecularweight PEG would be good candidates for a safe substitution of DEHP in soft PVC plasticized items.

Alternative copolymers of poly( $\varepsilon$-caprolactone) were also evaluated as a suitable substitute

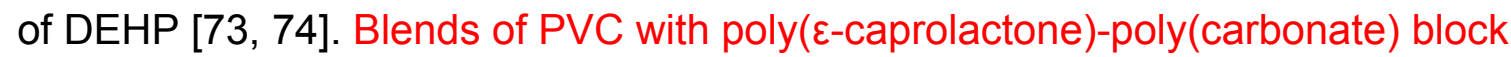
copolymers (PCL-PC) (Figure 4) have been investigated in order to assess the long-term behavior and release rate of the plasticizer in aqueous environments as well as the formation of degradation products. The plasticizer was found to be resistant to both hydrolysis and migration during the testing period at $37^{\circ} \mathrm{C}$. Migration of PCL-PC or its oligomers were observed after 70 days of immersion when a working temperature of $70^{\circ} \mathrm{C}$ was used. A large increase in the hydrolysis rate of PCL-PC and the complete migration of the plasticizer from the blend was observed when the aging temperature was raised to $100^{\circ} \mathrm{C}$. 
Most of the desirable properties associated with polymeric plasticizers are achieved by increasing their molecular weight. However, this could be partially unfavorable since it leads to increased viscosity of the materials and the longer processing time required to attain the blend. A decrease in plasticizer efficiency would also be expected and consequently a greater concentration of the plasticizer is required to achieve the desired plasticization of the polymer [75].

Branched and hyperbranched (HPB) polymers have been proposed as valid alternatives to linear polymeric plasticizers, to address the above concerns $[68,76,77]$. The unique structural characteristics of these bulk macromolecules provide them with the basic requirements for being considered promising alternative plasticizers. The spherical structures of HPB and/or the presence of several functional end-groups induced higher mobility to the plasticizer, thus improving its plasticization efficiency. At the same time the high molar mass and bulkiness of the branched polymer significantly hampers its own volatility and diffusivity compared to low molar mass plasticizers, thus drastically reducing any leaching concerns.

Choi and Kwak [76] reported the use of hyperbranched poly(ع-caprolactone) as a nonmigrating alternative plasticizer for phthalates in flexible PVC (Figure 4). The authors investigated the effects of some structural parameters on the efficiency of the analyzed plasticizer. The corresponding species constituted by shorter linear segments in its branching backbone and a large number of branches displayed a plasticization efficiency comparable to that of DEHP and excellent migration stability. 
Similar studies have been carried out on hyperbranched polyadipates $[68,77,78]$. Blends of poly(vinyl chloride) (PVC) and poly(butylene adipate) (PBA) with varying degrees of branching were analyzed with respect to migration resistance during aging in water, preservation of material properties, and thermal stability [78]. Highly branched poly(butyl adipate) (PBA) displayed poor miscibility with PVC and increased hydrophilicity, thus not representing a favorable plasticizer. Slightly branched PBA exhibited better plasticizing efficiency and migration resistance compared to the linear and highly branched polymers $[68,77]$.

Recently, considerable effort has been devoted to the exploitation of "green" plasticizers obtainable from renewable resources in order to reduce the negative impact of conventional PVC additives. Cyclic isosorbide derivatives in the polymeric form such as oligo(isosorbide adipate) and oligo(isosorbide suberate) have been investigated due to their structure similar to traditional phthalate plasticizers [79] (Figure 4). Preliminary investigation revealed a plasticizing behavior similar to that of the traditional phthalates, thus showing their potential as alternative PVC plasticizers.

\subsection{Chemical and physical modifications of PVC}

Along with the research of new and non-migrating plasticizers for replacing DEHP in PPVC formulations, several approaches have been developed to reduce DEHP migration. This strategy aims at extending the use of conventional P-PVC formulations in medical 
applications by introducing several chemical modifications to prevent plasticizer leaching. Various approaches proved to be effective in reducing plasticizer migration but at the same time introduced new chemicals into the P-PVC formulations that could be a cause for new concerns. The envisaged modifications could compromise the original properties of the material in a unpredictable manner and introduce further chemicals with questionable safety profiles. To date, surface modification of PVC by chemical and physical processes and covalent binding of the plasticizer to the PVC backbone are the most frequently investigated strategies.

\subsubsection{Surface Modification of PVC}

Surface modification of polymers has attracted great attention in biomaterial research because it can improve the biocompatibility of a polymer without compromising the mechanical properties. The biocompatibility of the polymer is almost exclusively affected by the chemistry of its surface. The safety profiles of the chemicals used for the coatings would be decisive for the final biocompatibility of the materials and their selection require thorough evaluation. The chemical and/or physical modification of the surface of PVC was developed mainly to reduce leaching of the plasticizer from PVC. The surface modification can be achieved by three main techniques, including surface coating, surface crosslinking through chemical and/or physical treatments, and alteration of surface characteristics by grafting. 


\subsection{1.a Surface Coating}

Coating a polymer surface with non-migrating material is a promising approach to reducing plasticizer migration from the polymer matrix, but usually these coatings are thick and often cause a reduced flexibility of the polymeric material. Instead, modification with thin coatings is an effective tool for overcoming leaching concerns without compromising the mechanical properties of the material. The most common techniques employed for their preparation are presented and discussed.

Chemical vapor deposition (CVD) is an excellent technique usually applied to coat structure as fibers or inner surface tubes with very thin films. However, the high temperature required for the process makes its application to polymers impossible. To overcome this drawback Breme et al. developed a new plasma-activated CVD able to coat the polymer with titanium-based layers without damaging the polymer itself [80]. This type of coating was found to prevent plasticizer leaching from PVC and improve bio- and bloodcompatibility of the polymer. The drawbacks of this methodology such as high cost and loss of transparency limits its application at present.

Surface coating of PVC has been alternatively performed with an organic-inorganic hybrid by a wet chemical process. $\alpha, \omega$-Triethoxysilane terminated poly(ethylene oxide) (PEO) was synthesized and used to produce hybrid coatings for PVC medical devices by means of the sol-gel approach [81]. The coating was found to greatly reduce the leaching of DEHP from PVC, but the authors stated that some processing parameters need to be optimized before any application to biomedical devices can be carried out. 
Heparin coatings have been also reported to reduce the leaching of DEHP from P-PVC. Karle et al. [82] did not show any release of DEHP from PVC tubes coated with heparin by the Cameda ${ }^{\circledR}$ method both in vitro and in vivo. However, the effects of such coating remain controversial. In a recent study, the barrier property to DEHP of three different surface coatings containing heparin in different forms (ionically or covalently linked) was examined through a Chandler loop model [83]. The results showed that the ionic heparin coatings displayed the best barrier property against DEHP extraction. The thin coatings were found to reduce DEHP leaching but do not provide a total and absolute barrier to DEHP migration when tested in vitro. The presence of MEHP was detected in the blood. and the amount was not lower in comparison to that found in blood derived from treatment with the uncoated control tubes. Sakurai et al. reported that the heparin-coated tubes did not reduce the extraction of DEHP after 30 min of circulation in vitro [84].

The use of $\beta$-cyclodextrin derivatives as PVC coatings was also envisaged in virtue of their known sequestering ability towards hydrophobic molecules and improved hydrophilicity [85]. This approach proved to be effective in improving the blood compatibility of the material but had negligible effects on the leaching of DEHP. A decrease in DEHP migration was observed only in media having low compatibility with DEHP, such as cottonseed oil. Comparative migration tests conducted with a powerful solvent for DEHP such as methanol showed a decrease of DEHP extraction only for a short period of treatment. 
Better results were observed by directly incorporating $\beta$-cyclodextrin derivatives into DEHP [86] and by in situ polymerization of $\beta$-cyclodextrin with vinyl chloride [87]. 2,3,6-perO-benzoyl- $\beta$-cyclodextrin (Bz- $\beta-C D$ ) was synthesized in order to introduce benzoyl groups in the $\beta$-cyclodextrin structure. The presence of benzoyl groups was envisaged to enhance the affinity between the $\beta$-cyclodextrin and DEHP by further stabilizing the inclusion complexes through $\pi-\pi$ interactions [86]. The DEHP incorporating Bz- $\beta-C D$ systems were employed in the preparation of PVC plastisol sheets and the systems were subjected to DEHP migration tests according to the International Organization for Standardization (ISO) method [86]. The results showed the marked efficacy of Bz- $\beta-C D$ in reducing the level of DEHP migration from P-PVC.

An effective reduction of DEHP leaching was also obtained by encapsulation of $\beta$ cyclodextrin in the polymer matrix by in situ polymerization with vinyl chloride [87]. $\beta$ cyclodextrin was opportunely modified with 3-(methacryloxy)propyl trimetoxysilane in order to introduce functional groups suitable for in situ polymerization with vinyl chloride. The covalent linkage between $\beta$-cyclodextrin derivative and PVC led to a good dispersion of the $\beta$-cyclodextrin in the matrix whose effect was considered to be responsible for the superior reduction effect on DEHP migration.

Although the last two strategies did not represent examples of surface modifications they have been reported to show both the controversial results obtained with $\beta-C D$ and how profoundly the site of action of $\beta-C D$ would affect the migratory behaviour of DEHP. 


\subsection{1.b. $\quad$ Surface crosslinking}

Surface crosslinking represents one of the most successful techniques to prevent DEHP migration from PVC medical devices. Crosslinking provides a tridimensional network of polymeric chains whose covalent linking determines a drastic decrease in their motion and flexibility. The crosslinked surface of a polymer behaves as a rigid barrier to the interfacial migration of molecules thus possibly intervening positively in the reduction of leaching of DEHP from P-PVC.

Crosslinking of a polymer surface can occur through physical treatments such as plasma or $\mathrm{y}$-irradiation, or through a previous chemical modification of the polymer surface followed by physical treatment.

Plasma treatment was successfully employed in prevention of DEHP leaching from the inner surface of narrow PVC tubes [88]. This versatile technique allows for the surface modification of the materials without changing their bulk properties. The effects of the argon plasma treatment on DEHP leaching have been investigated as a function of working parameters such as time of treatment, working pressure and applied voltage. The plasma treatments produced a considerable decrease in DEHP leachability and the migration was found to be hampered by extending the treatment time and decreasing the working pressure. The applied voltage did not significantly affect DEHP leaching.

Irradiation treatments with UV and y-rays have been extensively used to crosslink the surface of PVC sheets and tubes. These treatments have been applied both on unmodified PVC and on chemically modified PVC. 
Ito et al. [89] investigated the effect of optical irradiation (UV and visible light irradiation) on the leaching behavior of the plasticizer in PVC medical devices. Samples were irradiated by visible light using fluorescent lamps or by UV-rays using UV germicidal lamps with different exposure times. Migration tests carried out on the irradiated samples revealed that the treatments induced a decrease in the levels of DEHP migration, and the PVC products maintained their features, such as flexibility and stability.

The data have been interpreted by hypothesizing modifications of the superficial layers of PVC induced by UV irradiation. The significant leaching resistance of UV irradiated PVC devices was attributed to the oxidation and crosslinking occurred during the UV treatment as confirmed by ESCA and FT-IR analyses. The same authors investigated the effects of Y-ray irradiation on PVC sheets to verify the safety of the sterilization process $[49,50]$. They revealed a significant increase in the level of MEHP migration from the irradiated samples compared to that of the unsterilized control, thus posing some concerns about the safety of the irradiation process. The effects of irradiation on PVC and in general on polymeric materials is still quite controversial since they depend greatly on the energy of the incident light, irradiation doses and time of exposure. Both UV and $\mathrm{y}$ - irradiation may trigger crosslinking and/or degradation processes on the polymeric material and the additives by radical mechanism. PVC has been reported to undergo similar processes upon irradiation [90]. When PVC is gamma irradiated, crosslinking effects predominate at small doses (0-15 kGy), whereas at higher doses (25-100 kGy) main chain scissions are 
more pronounced [91]. The consequent formation of products of degradation could compromise the safety profile of the polymeric material in an unpredictable manner

The introduction of photoactive groups on the surface of PVC has been envisaged to protect the material from irradiation and favor surface crosslinking. The chemical modification has been commonly carried out by nucleophilic substitution of chlorine with suitably reactive photoactive groups. Sodium azide [92] and N,N-diethyl dithiocarbamate [93] have been conventionally used as nucleophilic species The substitution reactions were conducted in aqueous media in the presence of a phase transfer catalyst in order to confine the photoactive groups exclusively to the surface of the material. The photolytic instability of the introduced groups was then used to crosslink the PVC surfaces by UV radiation as shown in Figure 5.

(Figure 5)

The modifications were found to cause significant reduction of leaching of DEHP from the surface-modified PVC. A pronounced change in color of the crosslinked PVC samples treated with sodium azide raises several concerns about their possible use in medical applications. Indeed, the above behavior reveals the occurring of degradation processes at the surface of the irradiated materials interpreted with the dehydrochlorination of the polymer. 
Alternative surface crosslinking of PVC by chemical modification has been attempted by using sodium thiosulphate as well. The thiosulphate groups have been selectively introduced into the surface of PVC by performing the nucleophilic substitution of chlorine in aqueous media in the presence of tetrabutylammonium hydrogen sulphate as phase transfer agent [94]. The thiosulphate dianion can directly react with two chlorines from adjacent PVC chains or with one chlorine to afford the alkyl thiosulphate thus resulting in R-S-S-R and S-SO $-\mathrm{R}$ crosslinks (Figure 6a).

(Figure 6)

The surface crosslinking of PVC by thiosulphate was found to provoke harmful effects on organisms and was reported to cause hemolysis. Nevertheless, the above approach revealed a good DEHP migration resistance in the treated materials and the absence of side reactions. Sodium sulphide was alternatively used as a crosslinking agent to reduce the harmful effects of thiosulphate. [95]. The chemical crosslinking was carried out by the same chemical process encountered with azide and thiosulphate in order to obtain the treatment selectively on the surface without the assistance of irradiation technique (Figure 6b). The above modifications have been successfully applied to medical-grade PVC tubes and sheets. The treated samples showed a complete DEHP migration resistance when exposed for up to 30 days to hexane at $30^{\circ} \mathrm{C}$ and this ability was not altered by subjecting them to the sterilization process. 
The modified materials presented improved cytocompatibility in comparison to the samples treated with thiosulphate, but their preparations were usually accompanied by discoloration.

Poly(azido acrylates) have been alternatively used to coat P-PVC by UV crosslinking [96]. This type of treatment was found to induce crosslinking on both the surface and the bulk of the treated samples.

The formation of the crosslinked networks was confirmed by FT-IR spectroscopy analysis and was also considered responsible for the arrest of DEHP migration within the PVC polymer chain networks. This method appears promising in virtue of the simple preparation of the azide polymers and the effective barrier exerted against DEHP leaching.

\subsection{1.c. $\quad$ Surface Grafting}

Surface grafting is also an interesting approach to relieving P-PVC from plasticizer concerns. Its application allows for the formation of a stable covalent linkage between the grafted polymeric coating and the treated polymeric surface, thus ensuring it long-term stability. Covalent coatings are generally preferred to physically bound coatings due to their minor amount of chemicals released into the surrounding media. The nature of the covalent linkage greatly affects the stability of the polymeric coating since it determines its aptitude for degradation. Stable covalent linkages would pose fewer concerns about possible coating leaching and could be the first choice in the case of medical P-PVC. Most of the investigated surface modifications have been attempted with hydrophilic 
moieties in order to increase the biocompatibility of PVC with the interfaced organisms. This strategy has been currently achieved by two different methods classified as "graftingfrom" and "grafting-to" [97]. In the "grafting-from" approach an initiator molecule is immobilized on the polymer surface and allowed to react with suitable monomers to form a polymerized coating. The "grafting-to" process occurs when preformed end-functionalized polymer chains are covalently linked to the polymer surface.

The "grafting-to" method has been reported for the introduction of PEG moieties onto the PVC surface [98]. The modification was achieved by nucleophilic substitution of the chlorine atoms of P-PVC sheets or tubes with an excess of Na-PEG at $70^{\circ} \mathrm{C}$ according to the Williamson ether reaction. The conjugation of PEG chains onto the surface was found to significantly improve the blood compatibility of the P-PVC devices. The treated samples showed a pronounced decrease in DEHP migration in hydrophobic solvents such as petroleum ether, cottonseed oil and paraffin oil. This effect was attributed to the hydrophilic PEG surface that acted as a physical barrier against DEHP leaching. Further investigation is needed to assess the leaching behavior of the treated samples in aqueous media, thus reproducing the typical working conditions of P-PVC medical devices.

The grafting polymerization of different hydrophilic monomers onto a PVC surface was also achieved by a simple two-step grafting-from technique [99]. This procedure was based on a preliminary physical sorption of a hydrophobic free radical initiator on the PVC surface followed by radical polymerization of a suitable monomer in hydrophilic media. Different hydrophilic polymers were introduced on the PVC surface, such as poly(acrylic 
acid) (PAA), poly(2-hydroxyethyl methacrylate) (PHEMA), poly(dimethyl acrylamide) (PDMA), poly(dimethylaminoethyl methacrylate) (PDMAEMA), poly(2-hydroxyethyl acrylate) (PHEA) and poly(4-vinylpiridine) (P4VP). The thickness, roughness and chemical composition of the modified P-PVC surfaces were extensively investigated and the results showed the effective formation of a covalent coating of the hydrophilic polymers on the PVC surface. The authors presented their strategy as a valid method for reducing DEHP leaching from PVC but they did not demonstrate the above statement with objective data. Tests of plasticizer leaching from the treated samples are necessary to assess their reliability as alternatives to DEHP in medical applications.

\subsubsection{Plasticizers covalently bound to PVC}

The migration of DEHP from P-PVC can be achieved by the covalent linkage of the plasticizer to the hosting polymer. However, the broad modification of the bulk of the matrix is a major drawback of this strategy. The chemical treatments aimed at conjugating the plasticizer to the polymer could compromise its properties irreversibly and in an unpredictable manner. The wide use of chemicals could even worsen its safety profile. Navarro et al. published the covalent linkage of phthalates onto a PVC backbone upon suitable functionalization of the plasticizers [100]. Two different phthalate-containing thiol groups, namely di-(2-ethylhexyl) 4-mercaptophthalate (DOP-SH) and di(2-ethylhexyl) 5mercaptoisophthalate (iso DOP-SH) were successfully synthesized and used as nucleophiles in the substitution reaction of the chlorine atoms of PVC (Figure 7). The 
reactions were carried out in cyclohexanone at $60^{\circ} \mathrm{C}$. Under the selected conditions, no side reactions such as crosslinking or elimination were recorded.

The migration behavior of the plasticizers in the new materials was tested by extraction experiments using heptane at room temperature, and the results showed complete DEHP retention in the polymer.

Although most of the described findings were promising in reducing the leaching of plasticizers from P-PVC, the developed materials have been mainly tested with organic solvents, but no data regarding their behavior in aqueous media are available. The wide use of organic solvents reported in conventional migratory tests has revealed mainly the mobility of the plasticizer within the bulk of the polymer but data is lacking about the leaching extent in an aqueous environment. Further studies would be needed to obtain information regarding the leaching behavior of the developed materials in their envisaged working conditions.

(Figure 7)

A general overview of the advantages and disadvantages of chemical/physical treatments on P-PVC is summarized in Table 3.

(Table 3) 


\subsection{Alternative Polymers}

The possibility of using materials that do not require the use of plasticizers is undoubtedly an appealing approach to overcoming P-PVC concerns in medical applications. The use of PVC-free medical devices would limit the life-cycle hazard associated with PVC by reducing the emission of carcinogens such as vinyl chloride monomer and ethylene dichloride that are produced during PVC manufacturing, and also dioxins and hydrochloric acid formed during the combustion of PVC-medical waste in incinerators [101]. Modern technologies of PVC disposal and incineration are currently facing the above concerns but the exploitation of alternative polymers with favorable LCA profiles would be advisable. Substitution of PVC represents a challenging task due to the overall performance and low cost of this polymer. Any valid alternative material should provide similar performance at a comparable total system cost. The polymer needs to be inherently flexible, biocompatible and inert during the sterilization process.

Polymers considered to be suitable alternatives to PVC include poly(ethylene vinyl-coacetate) (EVA), polyolefins, silicones and polyurethanes (Figure 8).

(Figure 8) 
Although most of the selected materials perfectly matched the performance of P-PVC, their high cost is still a major limit to their exploitation. The safety profile of these polymers would also need to be tested properly since the toxicity data on humans, especially regarding their long-term effects, are lacking.

Relevant data of the described alternative polymers are summarized in Table 4.

(Table 4)

\subsubsection{Ethylene/vinyl acetate copolymers (EVA)}

EVA comprises copolymers of ethylene and vinyl acetate at various compositions of the monomeric units (Figure 8). The finely tuned combination of these components resulted in polymeric materials able to match the requirements of medical devices. Indeed they displayed prominent characteristics such as good flexibility, resistance to UV radiation, durability and ability to retain their structure properties over the time.

The inherent flexibility of EVA-based products depends on the vinyl acetate comonomer content and do not require the addition of softener agents. Accordingly they are well-suited for packaging and administration of lipophilic fluids. The material is approved for blood storage containers although it has been found to be more suitable for applications such as parenteral and enteral administration devices [102]. However, the release of plasticizers is 
also encountered with the commercial EVA-based devices since they are commonly assembled with connectors made in DEHP-PVC [103]. Kambia et al. investigated the exposure of children to DEHP from perfusion kits during long-term parenteral nutrition containing fat emulsions [103]. This study revealed both a constant release of DEHP from EVA bags with PVC-DEHP connections and a linear increase of DEHP leaching from outlet tubing. While EVA proved to be a promising alternative to PVC, the design of commercial medical products should also take into account the safety of the additional components.

\subsubsection{Polyolefins}

Polyolefins are currently the most widely used materials in the world for the fabrication of plastic items due to their relative low cost, durability and ease of processing. Mechanical properties of polyolefins are strictly dependent on the method and conditions of the polymerization process adopted, such as temperature, pressure and catalyst. The overall inertness of polyolefins make them suitable for applications requiring safety regulations such as food packaging and medical devices. Modifications of the polymer structure would provide the final material with the required flexibility to match the performances of P-PVC without the use of plasticizers. However, commercial polyolefins need the addition of stabilizers, such as anti-oxidants, to maintain their physical-chemical properties during processing, shelf life and use. The extent of migration of antioxidants and low molecular weight products from polyolefin should be of as much concern as the case of plasticizers 
in PVC. At present most studies have been devoted to the use of polyolefins in food packaging [104-106], while little information is available about the impact of these materials in medical fields [107-109]. The use of anti-oxidants in products intended for human-contact applications is nowadays strictly regulated by health issues and mandatory toxicity tests required by authorities and governments [110]. To address the above regulations, research is focusing on the exploitation of naturally occurring (biological) antioxidants and on limiting the leaching of additives through their proper immobilization in the polymeric matrix [110]. The formulation of safer antioxidants and/or reduction of leaching concerns would be a decisive step for the substitution of P-PVC with polyolefins. At present polyolefins are considered one of the most promising and recommended alternatives to P-PVC [21, 107, 108, 111-113], also in virtue of their competitive cost. The recent progress in metallocene catalyst technology has led to the development of even cheaper polyolefin materials [114]. By metallocene technology it is possible to obtain the polymer with a precise molecular architecture and a narrow molecular weight distribution. A fine control of the final properties of the material would allow for the preparation of polyolefins with improved flexibility without the use of toxic plasticizers. The major drawback of these materials is their petrochemical origin and concerns regarding their disposal.

The most common types of polyolefins are polyethylene and polypropylene. Polyethylene (PE) (Figure 8) is a thermoplastic polymer, inert, biocompatible and nontoxic [115]. It is used in a wide range of medical applications such as tubing, packaging films, 
sutures, blood collection and especially infusion lines. Co-extruded infusion lines of PE and P-PVC are currently available to limit the stiffness and opacity of PE. These devices combines the flexibility of P-PVC (outside of tubing) with the inertness of a polyethylene liner. However several studies have shown that co-extruded PVC-PE lines leached levels of DEHP comparable to those of pure PVC when submitted to extraction by lipid emulsions $[108,116-117]$. Loff et al. compared the performances of pure PE lines with those of pure PVC and co-extruded PVC-PE devices. The authors showed a safer use of this material, especially for applications in newborns [108]. These observations were corroborated subsequently by the same authors by comparing the toxicity of PVC with that of polyethylene medical devices. A lipid emulsion was administered to prepubertal rabbits through central IV lines continuously for 3 weeks either via PVC or polyethylene infusion systems. The PVC-administered group revealed multiple aberrations after exposure to the material while minor effects were detected for the PE treated-group [111].

Polypropylene (PP) (Figure 8) is a thermoplastic polymer with characteristics such as high clarity, good barrier properties and high flex resistance, which make it suitable for medical applications. Typical medical applications include tubing, bags and (especially in Europe) parenteral nutrition and dialysis films [115]. Its resistance to light and irradiation processes is relatively poor due to the presence of tertiary carbon atoms that facilitate the formation of radicals in the macromolecular backbone. Accordingly, polypropylene is added with antioxidants and radical scavengers to be stabilized from oxidative 
degradation. Nowadays the safety profiles and leaching aptitude of these additives are questions to be resolved for the safe use of polypropylene in medical applications.

\subsubsection{Polysiloxanes}

Polysiloxanes are a family of synthetic polymers whose main chain comprised the repetition of alternating silicon and oxygen atoms (Figure 8). In the lateral chain the silicon atoms are generally linked to organic groups (e.g., aliphatic and aromatic). Polysiloxanes are now one of the most thoroughly tested and widely used class of biomaterials due to their intrinsic biocompatibility and stability. Properties such as thermal stability, low surface tension and hydrophobicity have made polysiloxanes excellent materials in the production of catheters and other medical devices. They have been successfully applied in short- and long-dwelling catheters, dialysis machines and blood oxygenators [118]. Polysiloxane-based catheters used for parenteral nutrition have been shown to cause significantly less sepsis compared to alternative materials [119]. Patient compliance improved in comparison to that found with PVC catheters, due to the extended working life of the polysiloxane materials. However, a recent study proved the deterioration and device failure of gastrostomy polysiloxane catheters upon exposure to lipidic media [120].

In spite of the above finding, polysiloxane catheters and tubes are considered more stable than those made of PVC, and polysiloxane has been proposed as one of the most 
suitable alternative polymers for the production of medical devices characterized by a relatively short service life $[70,121]$.

Available data on the toxicity of polysiloxanes mainly regard the effects of the implantation of silicone devices or injection of the liquid precursors. The subcutaneous implantation of silicone preparations in mice has been shown to produce no significant changes in the weight of the most important organs and tissues [118].

The acute toxicity and the reproductive and developmental effects of polysiloxanes were tested in rats and rabbits in very dated works $[122,123]$. The results showed a lack of reproductive or teratogenic effects $[122,123]$ of several types of polydimethylsiloxane.

\subsubsection{Polyurethanes}

Polyurethanes (PU) are a family of synthetic polymers obtained by the polyaddition reaction of a di-isocyanate with a diol in the presence of a catalyst and other additives (Figure 8). By varying the nature of the two components it is possible to obtain polyurethanes characterized by different structural features responsible for their ultimate behavior ranging from hard to soft and flexible.

Similarly to polysiloxanes, polyurethanes possess greater durability than PVC, thus being excellent alternatives to PVC for the production of medical devices that are used for long service times such as percutaneuos endoscopy gastronomy tubes (PEG-tubes). Several papers have reported comparative studies on the durability of PEG-tubes made of PVC, 
PU and silicone. All the researchers agreed on the better performance of PU and silicones tubes with respect to their PVC counterparts, while conflicting data have been obtained by comparing the durability of polysiloxanes with that of PU [124-126].

Polyurethanes have been proposed as a valid alternative to P-PVC in blood bags mainly due to their low degree of thrombogenicity and ability to be sterilized by either ethylene oxide and ã-irradiation without any appreciable degradation [127].

The toxicity data of polyurethanes are nowadays limited and mainly related to the exposure and production of foams [128]. The potential health effects of polyurethanes in medical devices have not been fully assessed and would be worthy of further investigation in the light of their possible applications.

\section{Conclusions}

The substitution or modification of commercial P-PVC formulations in medical applications is increasingly demanded due to the risks associated with exposure to DEHP. The task is challenging, since a valid alternative material should be completely biocompatible and match the overall performance of P-PVC. Several approaches have been developed to improve the safety profile of the plasticizer and/or prevent its release from P-PVC medical devices. The alternatives described in this review are potentially suitable candidates for PPVC substitution. The chemical modification of PVC appears to be the least interesting approach, due to the questionable effects of the introduced chemicals both on its 
biocompatibility and the overall characteristics of the developed material. At present most of the strategies are devoted to decreasing the leaching of conventional plasticizers rather than improving their safety profiles. Indeed, the leaching behavior of the plasticizer cannot be universally optimized since is strongly affected by the chemical nature of the surrounding environment. Research on biocompatible plasticizers and/or materials, possibly of natural origin, is a safer approach to overcoming these P-PVC concerns. The exploitation of waste and renewable resources could be envisaged to overcome the economic drawbacks typically encountered in the production of materials from natural origin. However, all the proposed materials suffer from a significant lack of data regarding their toxicity and long-term effects on human health. These findings underline the need for a synergic interdisciplinary approach between clinicians, biologists and chemists to determine the best alternative to P-PVC for the production of safe and often long-term effective medical devices. Any proposed substitutes should be subject to a careful toxicological characterization including long-term studies, as well as to an accurate chemical-physical characterization to fully assess their compliance.

\section{Acknowledgments:}

Authors are grateful to Prof. Emo Chiellini, Dr. Ing. Dario Puppi and Mrs. Alison Frank for the critical reading of the manuscript. 


\section{References}

[1] Hakkarainen M. New PVC materials for medical applications-the release profile of PVC/polycaprolactone-polycarbonate aged in aqueous environments. Polymer Degradation and Stability. 2003;80:451-8.

[2] Global Business Intelligence (GBI) Research. Medical Polymers Market to 2015 Polyvinyl Chloride (PVC) Dominating the Medical Devices and Packaging Markets. In:Industry reports / Chemicals, pp. 116. http://gbiresearch.com/Report.aspx?ID=MedicalPolymers-Market-to-2015-Polyvinyl-Chloride-PVC-Dominating-the-Medical-Devices-andPackaging-Markets\&ReportType=Industry_Report\&coreindustry=ALL\&Title=Chemicals. Accessed November 2010.

[3] Zhao X, Courtney JM. Update on Medical Plasticized PVC. $1^{\text {st }}$ edition, Smithers Rapra, Shawbury, Shrewsbury, Shropshire (UK) 2009, 112 pp.

[4] Hakkarainen M, Aminlashgari N, Yin B. Biomass based plasticizers from liquefied waste paper. Angpanneforeningens Forskningsstiftelse (Aforsk). Reports within the forest industry research area, 2011, p.10. http://www.aforsk.se/images/pdf/10-065.pdf.

[5] Tickner JA, Rossi M, Haiama N, Lappe M, Hunt P. The use of di(2-ethylhexyl) phthalate in PVC medical devices: exposure, toxicity and alternatives. Lowell Center for Sustainable Production, University of Massachusetts, Lowell, 1999, p. 72.

http://www.sustainableproduction.org/downloads/DEHP\%20Full\%20Text.pdf. 
[6] Wypych G. Plasticizer types. In: Wypych G, editor. Handbook of plasticizers. Toronto, Ont.: ChemTec Publishing, 2004, pp. 7-73.

[7] Carlson K. Toxicity Review of Di(2-ethylhexyl) Phthalate (DEHP). United States Consumer Product Safety Commision (USCPS). editor Bethesda, MD, 2010, p. 329. http://www.cpsc.gov/ABOUT/Cpsia/toxicityDEHP.pdf.

[8] Latini G, De Felice C, Del Vecchio A, Barducci A, Ferri M, Chiellini F. Di-(2Ethylhexyl)Phthalate Leakage and Color Changes in Endotracheal Tubes after Application in High-Risk Newborns. Neonatology. 2009;95:317-23.

[9] Ferri M, Chiellini F, Pili G, Grimaldi L, Florio ET, Pili S, Cucci F, Latini G. Di-(2ethylhexyl)-phthalate migration from irradiated poly(vinyl chloride) blood bags for graft-vshost disease prevention. Int. J. Pharm.. 2012;430:86-8.

[10] Chiellini F, Ferri M, Latini G. Physical-chemical assessment of di-(2-ethylhexyl)phthalate leakage from poly(vinyl chloride) endotracheal tubes after application in high risk newborns. Int. J. Pharm.. 2011;409:57-61.

[11] Tickner JA, Schettler T, Guidotti T, McCally M, Rossi M. Health risks posed by use of Di-2-ethylhexyl phthalate (DEHP) in PVC medical devices: A critical review. Am. J. Ind. Med.. 2001;39:100-11.

[12] Miles-Richardson S. Toxicological profile for di(2-ethylhexyl)phthalate (DEHP). In: U.S. Department of Health and Human Services PHS, Agency for Toxic Substances and Disease Registry, editor. Atlanta, GA,2002, p. 336. http://www.atsdr.cdc.gov/toxprofiles/tp.asp?id=684\&tid=65. 
[13] Lefebvre P, Chinetti G, Fruchart JC, Staels B. Sorting out the roles of PPARa in energy metabolism and vascular homeostasis. J. Clin. Invest. 2005; 113: 571-580.

[14] Doull J, Cattley R, Elcombe C, Lake BG, Swenberg J, Wilkinson C, Williams G, van Gemert M. A cancer risk assessment of di(2-ethylhexyl)phthalate: application of the new U.S. EPA risk assessment guidelines. Regul. Toxicol. Pharmacol. 1999; 29:327-357. [15] Palmer C NA, Hsu M H, Griffin KJ, Raucy J L, Johnson E F. Peroxisome proliferator activated receptor- $\alpha$ expression in human liver. Mol. Pharmacol. 1998; 53: 14-22. [16] Melnick RL. Is Peroxisome Proliferation an Obligatory Precursor Step in the Carcinogenicity of Di(2-ethylhexyl)phthalate (DEHP)?. Environ. Health Persp. 2001; 109: 437-442.

[17] Ito $\mathrm{Y}$, Nakamura T, Yanagiba $\mathrm{Y}$, Ramdhan $\mathrm{DH}$, Yamagishi N, Naito H, Kamijima M, Gonzalez FJ, Nakajima T. Plasticizers May Activate Human Hepatic Peroxisome Proliferator-Activated Receptor a Less Than That of a Mouse but May Activate Constitutive Androstane Receptor in Liver. PPAR Res Hindawi Publishing Corporation. 2012 Article ID 201284, 11 pages doi:10.1155/2012/201284

[18] Latini G, Verrotti A, De Felice C. DI-2-Ethylhexyl Phthalate and Endocrine Disruption: A Review. Curr. Drug Targets: Immune, Endocr. Metab. Disord.. 2004;4:37-40. [19] Latini G. Monitoring phthalate exposure in humans. Clin. Chim. Acta. 2005;361:20-9. [20] Danschutter D, Braet F, Van Gyseghem E, Hachimi-Idrissi S, Van Bruwaene B, Moloney-Harmon P, Huyghens L.. Di-(2-ethylhexyl)phthalate and Deep Venous 
Thrombosis in Children: A Clinical and Experimental Analysis. Pediatrics. 2007;119:e742e53.

[21] von Rettberg H, Hannman T, Subotic U, Brade J, Schaible T, Waag KL, Loff S.. Use of Di(2-Ethylhexyl)Phthalate Containing Infusion Systems Increases the Risk for Cholestasis. Pediatrics. 2009;124:710-6.

[22] Green R, Hauser R, Calafat AM, Weuve J, Schettler T, Ringer S, S, Huttner K, Hu H.. Use of Di(2-ethylhexyl) Phthalate -Containing Medical Products and Urinary Levels of Mono(2-ethylhexyl) Phthalate in Neonatal Intensive Care Unit Infants. Environ Health Perspect. 2005;113, 1222-1225.

[23] Latini G, Ferri M, Chiellini F. Materials Degradation in PVC Medical Devices, DEHP Leaching and Neonatal Outcomes Curr. Med. Chem.. 2010;17:2979-89.

[24] Calafat AM, Needham LL, Silva MJ, Lambert G. Exposure to Di-(2-Ethylhexyl) Phthalate Among Premature Neonates in a Neonatal Intensive Care Unit. Pediatrics. 2004;113:e429-e34.

[25] Van Vliet EDS, Reitano EM, Chhabra JS, Bergen GP, Whyatt RM. A review of alternatives to di (2-ethylhexyl) phthalate-containing medical devices in the neonatal intensive care unit. J Perinatol. 2011;31:551-60.

[26] Rahman M, Brazel CS. Review:An assessment of traditional plasticizers and research trends for development of novel plasticizers. Prog. Polym. Sci. 2004; 29: 1223-1248. [27] Scientific Committee on Emerging and Newly-Identified Health Risks. Brussels (SCHENIR): European Commission. Opinion on the safety of medical devices containing 
DEHP plasticized PVC or other plasticizers on neonates and other groups possibly at risk, 2007, p.91,

http://ec.europa.eu/health/archive/ph_risk/committees/04_scenihr/docs/scenihr_o_014.pdf. [28] Kavlock R, Boekelheide K, Chapin R, Cunningham M, Faustman E, Foster P, P, Golub M, Henderson R, Hinberg I, Little R, Seed J, Shea K, Tabacova S, Tyl R, Williams P, Zacharewski T.. NTP Center for the Evaluation of Risks to Human Reproduction: phthalates expert panel report on the reproductive and developmental toxicity of diisodecyl phthalate. Reprod. Toxicol.. 2002;16:655-78.

[29] Shea KM, Committee on Environmental H. Pediatric Exposure and Potential Toxicity of Phthalate Plasticizers. Pediatrics. 2003;111:1467-74.

[30] Babich MA, Chen S-B, Greene MA, Kiss CT, Porter WK, Smith TP, Wind ML, Zamula WW.. Risk assessment of oral exposure to diisononyl phthalate from children's products. Regul. Toxicol. Pharm. 2004;40:151-67.

[31] Deyo JA. Carcinogenicity and chronic toxicity of di-2-ethylhexyl terephthalate (DEHT) following a 2-year dietary exposure in Fischer 344 rats. Food Chem. Toxicol.. 2008;46:990-1005.

[32] Barber ED, Fox JA, Giordano CJ. Hydrolysis, absorption and metabolism of di(2ethylhexyl) terephthalate in the rat. Xenobiotica. 1994;24:441-50. 
[33] Mitchell AM, Lhuguenot J-C, Bridges JW, Elcombe CR. Identification of the proximate peroxisome proliferator(s) derived from Di(2-ethylhexyl) phthalate. Toxicol. Apll. Pharm. 1985;80:23-32.

[34] Tomita I, Nakamura Y, Aoki N, Inui N. Mutagenic/carcinogenic potential of DEHP and MEHP. Environ Health Perspect 1982;45:119-25.

[35] Dalgaard M, Nellemann C, Lam HR, Sørensen IK, Ladefoged O. The acute effects of mono(2-ethylhexyl)phthalate (MEHP) on testes of prepubertal Wistar rats. Toxicol. Lett.. 2001;122:69-79.

[36] Gray LE, Ostby J, Furr J, Price M, Veeramachaneni DNR, Parks L. Perinatal Exposure to the Phthalates DEHP, BBP, and DINP, but Not DEP, DMP, or DOTP, Alters Sexual Differentiation of the Male Rat. Toxicol. Lett. 2000;58:350-65.

[37] Faber WD, Deyo JA, Stump DG, Navarro L, Ruble K, Knapp J. Developmental toxicity and uterotrophic studies with di-2-ethylhexyl terephthalate. Birth Defects Res. B Dev. Reprod. Toxicol.. 2007;80:396-405.

[38] Faber WD, Deyo JA, Stump DG, Ruble K. Two-generation reproduction study of di-2ethylhexyl terephthalate in Crl:CD rats. Birth Defects Res. B Dev. Reprod. Toxicol.. 2007;80:69-81.

[39] Maag J, Lassen C, Brandt UK, Kjølholt J, Molander L, Mikkelsen SH. Identification and assessment of alternatives to selected phthalates. COWI: Environmental Project No. 13412010 Miljøprojekt. Copenhagen: Danish EPA, 2010,p.288. 
http://www2.mst.dk/udgiv/publications/2010/978-87-92708-00-7/pdf/978-87-92708-014.pdf

[40] Barber ED, Topping DC. Subchronic 90-day oral toxicology of Di(2-ethylhexyl) terephthalate in the rat. Food Chem. Toxicol. 1995;33:971-8.

[41] L.G. Krauskopf and A. Godwin. Plasticizers. In: CE Wilkes, Summers JW, Daniels CA, editors. PVC Handbook. Carl Hanser Verlag, Munich, 2005, Chapter 5, pp. 173-199 [42] U.S. EPA High Production Volume (HPV) Chemicals Challenge Program. Assessment of data availability and test plan for acetyl tributyl citrate (ATBC), 2003, p.132, http://www.epa.gov/HPV/pubs/summaries/acetlcit/c15025.pdf.

[43] Johnson WJ. Final Report on the Safety Assessment of Acetyl Triethyl Citrate, Acetyl Tributyl Citrate, Acetyl Trihexyl Citrate, and Acetyl Trioctyl Citrate. Int. J. Toxicol.. $2002 ; 21: 1-17$.

[44] Hull EH. Medical articles and method. US 4,710,532, Morflex Chemical Co, 1987. [45] Hull EH. Citrate esters and methods. US Patent 4824893, Morflex Chemical Company, Inc, 1989.

[46] Hillyer CD. Blood banking and transfusion medicine. 2nd Ed.Churchill Livingstone. Elsevier, Philadelphia, 2007, p.887.

[47] Ito R, Miura N, Iguchi H, Nakamura H, Ushiro M, Wakui N, Nakahashi K, Iwasaki Y, Saito K, Suzuki T, Nakazawa H. Determination of tris(2-ethylhexyl)trimellitate released from PVC tube by LC-MS/MS. Int. J. Pharm.. 2008;360:91-5. 
[48] Kambia K, Dine T, Azar R, Gressier B, Luyckx M, Brunet C. Comparative study of the leachability of di(2-ethylhexyl) phthalate and tri(2-ethylhexyl) trimellitate from haemodialysis tubing. Int. J. Pharm.. 2001;229:139-46.

[49] Ito R, Seshimo F, Miura N, Kawaguchi M, Saito K, Nakazawa H. Effect of sterilization process on the formation of mono(2-ethylhexyl)phthalate from di(2-ethylhexyl)phthalate. J. Pharm. Biomed. Anal.. 2006;41:455-60.

[50] Ito R, Miura N, Ushiro M, Kawaguchi M, Nakamura H, Iguchi H, Ogino J, Oishi M, Wakui N,. Iwasaki Y, Saito K, Nakazawa H.. Effect of gamma-ray irradiation on degradation of di(2-ethylhexyl)phthalate in polyvinyl chloride sheet. Int. J. Pharm. 2009;376:213-8.

[51] ter Veld MGR, Schouten B, Louisse J, van Es DS, van der Saag PT, Rietjens IMCM, et al. Estrogenic Potency of Food-Packaging-Associated Plasticizers and Antioxidants As Detected in ERÎt and ERÎ Reporter Gene Cell Lines. J. Agr. Food Chem.. 2006;54:440716.

[52] Kambia K, Dine T, Gressier B, Dupin-Spriet T, Luyckx M, Brunet C. Evaluation of the direct toxicity of trioctyltrimellitate (TOTM), di(2-ethylhexyl) phthalate (DEHP) and theirhydrolysis products on isolated rat hepatocytes. Int. J. Artif. Organs. 2004;27:971. [53] David RM, Lockhart LK, Ruble KM. Lack of sensitization for trimellitate, phthalate, terephthalate and isobutyrate plasticizers in a human repeated insult patch test. Food Chem. Toxicol.. 2003;41:589-93. 
[54] Rahman M. Effectiveness of imidazolium, ammonium and phosphonium based ionic liquids as plasticizers for poly(vinyl chloride) and poly(methyl methacrylate). University of Alabama: Tuscaloosa AL 2004, 220 p..

[55] Rahman M, Brazel CS. Ionic liquids: New generation stable plasticizers for poly(vinyl chloride). Polym. Degrad. Stabil. 2006;91:3371-82.

[56] Kastner J, Cooper DG, Marić M, Dodd P., Yargeau V. Aqueous leaching of di-2ethylhexyl phthalate and "green" plasticizers from poly(vinyl chloride). Sci Total Environ. 2012; 432: 357-364

[57] Crespo JE, Balart R, Sanchez L, López J. Substitution of di(2-ethylhexyl) phthalate by di(isononyl) cyclohexane-1,2-dicarboxylate as a plasticizer for industrial vinyl plastisol formulations. J. Appl. Polym. Sci.. 2007;104:1215-20.

[58] Burgos N, Jiménez A. Degradation of poly(vinyl chloride) plasticized with nonphthalate plasticizers under sterilization conditions. Polym. Degrad. Stabil. 2009;94:14738.

[59] Manfredini M, Atzei D, Elsener B, Marchetti A, Rossi A. Degradation of plasticized PVC for biomedical disposable device under soft x-ray irradiation. Surf. Interface Anal. 2003;35:294-300.

[60] Vinhas GM, Souto Maior RM, de Almeida YMB. Radiolytic degradation and stabilization of poly(vinyl chloride). Polym. Degrad. Stabil.. 2004;83:429-33. 
[61] Atzei D, Elsener B, Manfredini M, Marchetti A, Malagoli M, Galavotti F, et al.

Radiation-induced migration of additives in PVC-based biomedical disposable devices Part 2. Surface analysis by XPS. Surf. Interface Anal.. 2003;35:673-81.

[62] Wadey BL. An innovative plasticizer for sensitive applications. J. Vinyl Addit. Technol.. 2003;9:172-6.

[63] Arendt WD, Lang J. New benzoate plasticizers for polyvinyl chloride: Introduction and performance example. J. Vinyl Addit. Technol. 1998;4:184-8.

[64] Gil N, Saska M, Negulescu I. Evaluation of the effects of biobased plasticizers on the thermal and mechanical properties of poly(vinyl chloride). J. Appl. Polym. Sci.. 2006;102:1366-73.

[65] Shah BL, Shertukde VV. Effect of plasticizers on mechanical, electrical, permanence, and thermal properties of poly(vinyl chloride). J. Appl. Polym. Sci.. 2003;90:3278-84. [66] Sunny MC, Ramesh P, George KE. Use of Polymeric Plasticizers in Polyvinyl Chloride to Reduce Conventional Plasticizer Migration for Critical Applications. J. Elastom. Plast.. 2004;36:19-31.

[67] Soong SY, Cohen RE, Boyce MC. Polyhedral oligomeric silsesquioxane as a novel plasticizer for poly(vinyl chloride). Polymer. 2007;48:1410-8.

[68] Lindström A, Hakkarainen M. Environmentally friendly plasticizers for poly(vinyl chloride)_-Improved mechanical properties and compatibility by using branched poly(butylene adipate) as a polymeric plasticizer. J. Appl. Polym. Sci. 2006;100:2180-8. 
[69] Wang Q, Storm BK. Migration of Additives from Poly(vinyl chloride) (PVC) Tubes into Aqueous Media. Macromol. Symp. 2005;225:191-204.

[70] Subotic U, Hannmann T, Kiss M, Brade J, Breitkopf K, Loff S. Extraction of the Plasticizers Diethylhexylphthalate and Polyadipate From Polyvinylchloride Nasogastric Tubes Through Gastric Juice and Feeding Solution. J. Pediatr. Gastr. Nutr. 2007;44:71-6. [71] Penco M, Sartore L, Bignotti F, Rossini M, D'Amore A, Fassio F. Binary blends based on poly(vinyl chloride) and multi-block copolymers containing poly(ع-caprolactone) and poly(ethylene glycol) segments. Macromol. Symp.. 2002;180:9-22.

[72] Ferruti P, Mancin I, Ranucci E, De Felice C, Latini G, Laus M.

Polycaprolactone/Poly(ethylene glycol) Multiblock Copolymers as Potential Substitutes for Di(ethylhexyl) Phthalate in Flexible Poly(vinyl chloride) Formulations. Biomacromolecules. 2003;4:181-8.

[73] Ferruti P, Latini G. Polymeric materials of the polyester-carbonate family and reactions for the formation of such materials 2000, English patent, EP 1036806 A2, EVC Compounds (Italy) S.P.A..

[74] Ferruti P, Latini G. Polymeric materials with a polyvinyl chloride and polyestercarbonate base 2000, English patent, EP1036816 A1, EVC Compounds (Italy) S.P.A. [75] Biesiada K, Fisch M, Peveler R. Propanedol-derived polyesters as PVC plasticizers. 2000, US 6,111,004. Velsicol Chemical Corp,.

[76] Choi J, Kwak S-Y. Hyperbranched Poly(e-caprolactone) as a Nonmigrating Alternative Plasticizer for Phthalates in Flexible PVC. Environ. Sci. Technol 2007;41:3763-8. 
[77] Lindström A, Hakkarainen M. Migration resistant polymeric plasticizer for poly(vinyl chloride). J J. Appl. Polym. Sci. 2007;104:2458-67.

[78] Lindström A, Hakkarainen M. Designed Chain Architecture for Enhanced Migration Resistance and Property Preservation in Poly(vinyl chloride)/Polyester Blends. Biomacromolecules. 2007;8:1187-94.

[79] Yin B, Hakkarainen M. Oligomeric isosorbide esters as alternative renewable resource plasticizers for PVC. J. Appl. Polym. Sci.. 2011;119:2400-7.

[80] Breme F, Buttstaedt J, Emig G. Coating of polymers with titanium-based layers by a novel plasma-assisted chemical vapor deposition process. Thin Solid Films. 2000;377378:755-9.

[81] Messori M, Toselli M, Pilati F, Fabbri E, Fabbri P, Pasquali L, Nannarone S.

Prevention of plasticizer leaching from PVC medical devices by using organic-inorganic hybrid coatings. Polymer. 2004;45:805-13.

[82] Karle VA, Short BL, Martin GR, Bulas DI, Getson PR, Luban NLC, O'Brien AM, Rubin

RJ.. Extracorporeal membrane oxygenation exposes infants to the plasticizer, di(2ethylhexyl)phthalate. Crit. Care Med.. 1997;25:696-703.

[83] Hildebrand SL, Lehmann H-D, Wodarz R, Ziemer G, Wendel H. PVC-plasticizer DEHP in medical products: do thin coatings really reduce DEHP leaching into blood? Perfusion. 2005;20:351-7. 
[84] Sakurai H, Maeda M, Miyahara K, Nakayama M, Murayama H, Hasegawa H, Hayakawa M, Sugiura T, Kamikura K.. Extraction of di-2-ethylhexyl phthalate from a cardiopulmonary bypass circuit. Kyobu Geka. 2001;54:471-4.

[85] Zhao X, Courtney JM. Surface modification of polymeric biomaterials: Utilization of cyclodextrins for blood compatibility improvement. J. Biomed. Mater. Res. A. 2007;80A:539-53.

[86] Yu BY, Chung JW, Kwak S-Y. Reduced Migration from Flexible Poly(vinyl chloride) of a Plasticizer Containing b-Cyclodextrin Derivative. Environ. Sci. Technol.. 2008;42:7522-7. [87] Chung JW, Kim SH, Jung SJ, Kwak S-Y. Encapsulation of b-cyclodextrin by in situ polymerization with vinyl chloride leading to suppressing the migration of endocrine disrupting phthalate plasticizer. Eur. Polym. J.. 2009;45:2164-71.

[88] Xiao Qiong W, Xue Hua L, Gui Shi L. Prevention of Plasticizer Leaching From the Inner Surface of Narrow Polyvinyl Chloride Tube by DC Glow Discharge Plasma. IEEE T. Plasma Sci.. 2010;38:3152-5.

[89] Ito R, Seshimo F, Haishima Y, Hasegawa C, Isama K, Yagami T, Nakahashi K, Yamazahi H, Inoue K, Yoshimura Y, Saito K, Tsuchiya T, Nakazawa H. Reducing the migration of di-2-ethylhexyl phthalate from polyvinyl chloride medical devices. Int. J. Pharm.. 2005;303:104-12.

[90] Baccaro S, Brunella V, Cecilia A, Costa L. y irradiation of poly(vinyl chloride) for medical applications. Nucl. Instrum. Meth. B. 2003; 208: 195-198. 
[91] da Silva FF, da S. Aquino KA, Arau'jo E.S. Effects of gamma irradiation on poly(vinyl chloride)/polystyrene blends:Investigation of radiolytic stabilization and miscibility of the mixture. Polym. Degrad. Stabil. 2008; 93: 2199-2203.

[92] Jayakrishnan A, Sunny MC. Phase transfer catalysed surface modification of plasticized poly(vinyl chloride) in aqueous media to retard plasticizer migration. Polymer. $1996 ; 37: 5213-8$.

[93] Lakshmi S, Jayakrishnan A. Photocross-linking of dithiocarbamate-substituted PVC reduces plasticizer migration. Polymer. 1998;39:151-7.

[94] Lakshmi S, Jayakrishnan A. Synthesis, surface properties and performance of thiosulphate-substituted plasticized poly(vinyl chloride). Biomaterials. 2002;23:4855-62. [95] Lakshmi S, Jayakrishnan A. Properties and performance of sulfide-substituted plasticized poly(vinyl chloride) as a biomaterial. J. Biomed. Mater. Res., Part B. 2003;65B:204-10.

[96] Reddy NN, Mohan YM, Varaprasad K, Ravindra S, Vimala K, Raju KM. Surface treatment of plasticized poly(vinyl chloride) to prevent plasticizer migration. J. Appl. Polym. Sci. 2010;115:1589-97.

[97] Zhao B, Brittain WJ. Polymer brushes: surface-immobilized macromolecules. Prog. Polym. Sci. 2000;25:677-710.

[98] Lakshmi, Jayakrishnan. Migration resistant, blood-compatible plasticized polyvinyl chloride for medical and related applications. Artif. Organs. 1998;22:222-9. 
[99] McGinty KM, Brittain WJ. Hydrophilic surface modification of poly(vinyl chloride) film and tubing using physisorbed free radical grafting technique. Polymer. 2008;49:4350-7. [100] Navarro R, Perez Perrino M, Gomez Tardajos M, Reinecke H. Phthalate Plasticizers Covalently Bound to PVC: Plasticization with Suppressed Migration. Macromolecules. 2010;43:2377-81.

[101] Wagner JC, Green AES. Correlation of chlorinated organic compound emissions from incineration with chlorinated organic input. Chemosphere. 1993;26:2039-54. [102] Toxics Use Reduction Institute (TURI) publications. DEHP. In: Five chemicals alternatives assessment study.Toxics Use Reduction Institute (TURI). Lowell MA: Univ. of Massachusetts,2006,p.92.http://www.turi.org/About/Library/TURI_Publications/2006_Five_ Chemicals_Alternatives_Assessment_Study/Final-Report/Chapter-7.-DEHP. [103] Kambia K, Dine T, Gressier B, Bah S, Germe AF, Luyckx M, Brunet C, Michaud L, Goltrand F.. Evaluation of childhood exposure to di(2-ethylhexyl) phthalate from perfusion kits during long-term parenteral nutrition. Int. J. Pharm.. 2003;262:83-91.

[104] Dopico-García M, López-Vilariñó J, Gonzalez-Rodríguez M. Antioxidant Content of and Migration from Commercial Polyethylene, Polypropylene, and Polyvinyl Chloride Packages. J. Agr. Food Chem.. 2007;55:3225-31.

[105] Dopico-García M, López-Vilariñó J, Gonzalez-Rodríguez M. Determination of antioxidant migration levels from low-density polyethylene films into food simulants. J. Chromatogr., A. 2003;1018:53-62. 
[106] Tovar L, Salafranca J, Sanchez C, Nerin C. Migration Studies To Assess the Safety in Use of a New Antioxidant Active Packaging. J. Agr. Food Chem.. 2005;53:5270-5. [107] Marcato B, Guerra S, Vianello M, Scalia S. Migration of antioxidant additives from various polyolefinic plastics into oleaginous vehicles. Int. J. Pharm.. 2003;257:217-25. [108] Loff S, Subotic U, Reinicke F, Wischmann H, Brade J. Extraction of Di-ethylhexylphthalate from Perfusion Lines of Various Material, Length and Brand by Lipid Emulsions. J. Pediatr. Gastr. Nutr.. 2004;39:341-5.

[109] Sautou-Miranda V, Brigas F, Vanheerswynghels S, Chopineau J. Compatibility of paclitaxel in $5 \%$ glucose solution with ECOFLAC $\otimes$ low-density polyethylene containersstability under different storage conditions. Int. J. Pharm.. 1999;178:77-82.

[110] Al-Malaika S, Albertsson A-C. Perspectives in Stabilisation of Polyolefins Long Term Properties of Polyolefins. In: Albertsson AC. ,editor, Long Term Properties of Polyolefins. Berlin/Heidelberg: Springer , 2004, p.121-150.

[111] Loff PDS, Subotic U, Oulmi-Kagermann J, Kränzlin B, Reinecke M-F, Staude C. Diethylhexylphthalate Extracted by Typical Newborn Lipid Emulsions From Polyvinylchloride Infusion Systems Causes Significant Changes in Histology of Rabbit Liver. JPen-Parenter. Enter.. 2007;31:188-93.

[112] Loff S, Hannmann T, Subotic U, Reinecke F-M, Wischmann H, Brade J. Extraction of Diethylhexylphthalate by Home Total Parenteral Nutrition From Polyvinyl Chloride Infusion Lines Commonly Used in the Home. J. Pediatr. Gastr. Nutr. 2008;47:81-6 10.1097/MPG.0b013e318164d933. 
[113] Sampson J, de Korte D. DEHP-plasticised PVC: relevance to blood services. Transfusion Med. 2011;21:73-83.

[114] Sunny MC, Ramesh P, Mohanan PV, George KE. Metallocene based polyolefin: a potential candidate for the replacement of flexible poly (vinyl chloride) in the medical field. Polym. Advan. Technol.. 2010;21:621-31.

[115] Sastri V. Plastics in Medical Devices. Properties, requirements and applications. 1st Ed. PDL Handbooks Series. Elsevier, William Andrew Amsterdam; Boston, 2010, p.271. [116] Bagel-Boithias S, Sautou-Miranda Vr, Bourdeaux D, Tramier V, Boyer A, Chopineau J. Leaching of diethylhexyl phthalate from multilayer tubing into etoposide infusion solutions. Am. J. Health Syst. Ph.. 2005;62:182-8.

[117] Bourdeaux D, Sautou-Miranda Vr, Bagel-Boithias S, Boyer A, Chopineau J. Analysis by liquid chromatography and infrared spectrometry of di(2-ethylhexyl)phthalate released by multilayer infusion tubing. J. Pharm. Biomed. Anal.. 2004;35:57-64.

[118] National Toxicology Program. Department of Health and Human Services. Report MM89050 (Abstract). The Immunotoxicity of Silicone. 2012.

http://ntp.niehs.nih.gov/index.cfm?objectid=03E82B79-FF50-8E65-2104927658C2EF01 [119] Mitchell A, Atkins S, Royle GT, Kettlewell MGW. Reduced catheter sepsis and prolonged catheter life using a tunnelled silicone rubber catheter for total parenteral nutrition Brit. J. Surg. 1982;69:420-22.

[120] Trudel J, Gauderer MWL, Drews MJ, LaBerge M. Lipid uptake by silicone enteral access feeding devices. J. Pediatr. Surg.. 1998;33:880-4. 
[121] Mair AA, Mair EA. Can plastic tracheotomy tubes harm baby boys? Otolaryngol. Head Neck Surg. 2009;140:13-4.

[122] Kennedy GLJ, Keplinger ML, Calandra JC. Reproductive, teratologic, and mutagenic studies with some polydimethylsiloxanes. J. Toxicol. Environ. Health. 1976;1:909-20. [123] Hobbs EJ, Fancher OE, Calandra JC. Effect of selected organopolysiloxanes on male rat and rabbit reproductive organs. Toxicol. Apll. Pharm.. 1972;21:45-54. [124] Björling G, Axelsson S, Johansson U-B, Lysdahl M, Markström A, Schedin U, Aune RE, Frostell C, Karlsson S. Clinical Use and Material Wear of Polymeric Tracheostomy Tubes. The Laryngoscope. 2007;117:1552-9.

[125] Sartori S, Trevisani L, Nielsen I, Tassinari D, Ceccotti P, Abbasciano V. Longevity of silicone and polyurethane catheters in long-term enteral feeding via percutaneous endoscopic gastrostomy. Aliment. Pharm. Ther.. 2003;17:853-6.

[126] Blacka J, Donoghue J, Sutherland M, Martincich I, Mitten-Lewis S, Morris P, Meredith G.. Dwell time and functional failure in percutaneous endoscopic gastrostomy tubes: a prospective randomized-controlled comparison between silicon polymer and polyurethane percutaneous endoscopic gastrostomy tubes. Aliment. Pharm. Ther.. 2004;20:875-82.

[127] Simmchen J, Ventura R, Segura J. Progress in the Removal of Di-(2-Ethylhexyl)Phthalate as Plasticizer in Blood Bags. Transfusion Med.. 2012;26:27-37. 
[128] Cragg ST. Polyurethanes, Miscellaneous Organic Polymers, and Silicones. In:

Bingham E, Cohrssen, editors. Patty's Toxicology, John Wiley \& Sons Inc, 2012, Chapter 71, pp. $999-1038$ 
Captions to figures

Figure 1. Different flexibility grades of commercial PVC-type materials according to their different plasticizer content and related medical applications.

Figure 2. Outline of current approaches for the substitution of DEHP plasticized PVC.

Figure 3. Chemical structures of common low molecular weight plasticizers selected as alternative to DEHP.

Figure 4. Chemical structure of polymeric plasticizers investigated as alternatives to DEHP Figure 5. Scheme of the surface crosslinking of PVC induced by UV irradiation after nucleophilic substitution of chlorine atoms of PVC with(a) sodium azide or (b) N,N-diethyl dithiocarbamate.

Figure 6. Scheme of the surface crosslinking of PVC induced by UV irradiation after nucleophilic substitution of chlorine atoms of PVC with (a) sodium thiosulphate or (b) sodium sulphide.

Figure 7. Scheme of the nucleophilic substitution of chlorine atoms of PVC with di(2ethylhexyl) 4-mercaptophthalate and di(2-ethylhexyl) 5-mercaptoisophthalate Figure 8. Chemical structure of common polymers studied as alternative to P-PVC 